\title{
GLUING DELAUNAY ENDS TO MINIMAL $n$-NOIDS USING THE DPW METHOD
}

\author{
MARTIN TRAIZET
}

Abstract: we construct constant mean curvature surfaces in euclidean space by gluing $n$ half Delaunay surfaces to a non-degenerate minimal n-noid, using the DPW method.

\section{INTRODUCTION}

In [3], Dorfmeister, Pedit and $\mathrm{Wu}$ have shown that surfaces with non-zero constant mean curvature (CMC for short) in euclidean space admit a Weierstrass-type representation, which means that they can be represented in terms of holomorphic data. This representation is now called the DPW method. In [14], we used the DPW method to construct CMC $n$-noids: genus zero, CMC surfaces with $n$ ends Delaunay type ends. These $n$-noids can be described as a unit sphere with $n$ half Delaunay surfaces with small necksizes attached at prescribed points. They had already been constructed by Kapouleas in [8] using PDE methods.

In the case $n=3$, Alexandrov-embedded CMC trinoids have been classified by Große Brauckman, Kusner and Sullivan in [6]. In particular, equilateral CMC trinoids form a 1-parameter family, parametrized on an open interval. On one end, equilateral trinoids degenerate like the examples described above: they look like a sphere with 3 half Delaunay surfaces with small necksizes attached at the vertices of a spherical equilateral triangle. On the other end, equilateral trinoids limit, after suitable blow-up, to a minimal 3-noid: a genus zero minimal surface with 3 catenoidal ends (see Figure 1).

It seems natural to ask if one can generalize this observation and construct CMC $n$-noids by gluing half Delaunay surfaces with small necksizes to a minimal $n$-noid. This is indeed the case, and has been done by Mazzeo and Pacard in [10] using PDE methods. In this paper, we propose a quite simple and natural DPW potential to construct these examples. We prove:

Theorem 1. Let $n \geq 3$ and let $M_{0}$ be a non-degenerate minimal n-noid. There exists a smooth family of CMC surfaces $\left(M_{t}\right)_{0<|t|<\epsilon}$ with the following properties:

(1) $M_{t}$ has genus zero and $n$ Delaunay ends.

(2) $\frac{1}{t} M_{t}$ converges to $M_{0}$ as $t \rightarrow 0$.

(3) If $M_{0}$ is Alexandrov-embedded, all ends of $M_{t}$ are of unduloid type if $t>0$ and of nodoid type if $t<0$. Moreover, $M_{t}$ is Alexandrov-embedded if $t>0$.

Non-degeneracy of a minimal $n$-noid will be defined in Section 2, The two surfaces $M_{t}$ and $M_{-t}$ are geometrically different: if $M_{t}$ has an end of unduloid type then the corresponding end of $M_{-t}$ is of nodoid type. See Proposition 5 for more details. Of course, a minimal $n$ noid is never embedded if $n \geq 3$ so the surfaces $M_{t}$ are not embedded. Alexandrov-embedded minimal $n$-noids whose ends have coplanar axes have been classified by Cosin and Ros in [2], 

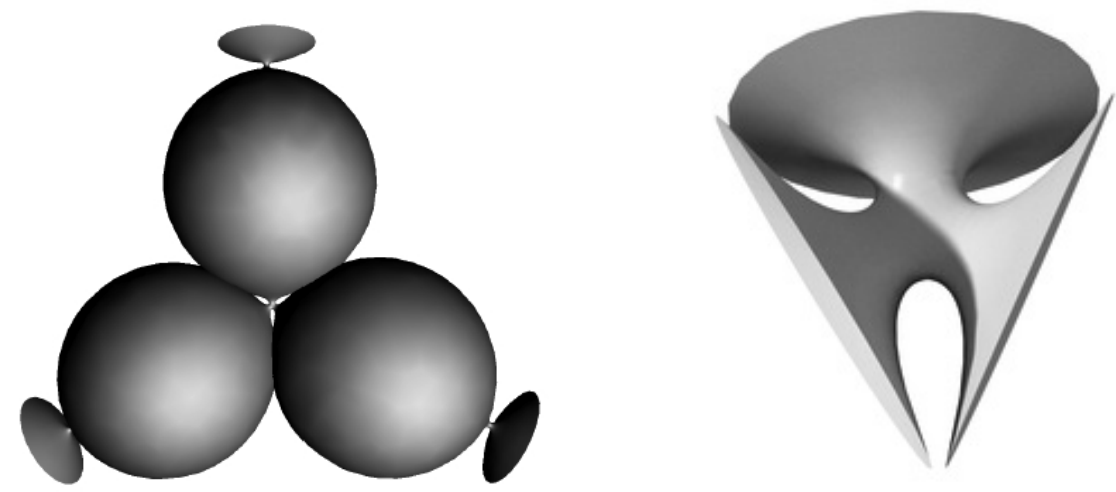

Figure 1. A CMC 3-noid (left, image by N. Schmitt [12]) and a minimal 3-noid (right). There is a tiny "copy" of the minimal 3-noid at the center of the CMC 3-noid.

and Alexandrov-embedded CMC $n$-noids whose ends have coplanar axes have been classified by Große-Brauckmann, Kusner and Sullivan in [7.

As already said, these surfaces have already been constructed in [10]. Our motivation to construct them with the DPW method is to answer the following questions:

(1) How can we produce a DPW potential from the Weierstrass data $(g, \omega)$ of the minimal $n$-noid $M_{0}$ ?

(2) How can we prove, with the DPW method, that $\frac{1}{t} M_{t}$ converges to $M_{0}$ ?

The answer to Question 2 is Theorem 4 in Section 4, a general blow-up result in the context of the DPW method. In [15], we use the DPW method to construct higher genus CMC surfaces with small necks. Theorem 4 is used to ensure that the necks have asymptotically catenoidal shape.

\section{NON-DEGENERATE MINIMAL $n$-NOIDS}

A minimal $n$-noid is a complete, immersed minimal surface in $\mathbb{R}^{3}$ with genus zero and $n$ catenoidal ends. Let $M_{0}$ be a minimal $n$-noid and $(\Sigma, g, \omega)$ its Weierstrass data. This means that $M_{0}$ is parametrized on $\Sigma$ by the Weierstrass Representation formula:

$$
\psi(z)=\operatorname{Re} \int_{z_{0}}^{z}\left(\frac{1}{2}\left(1-g^{2}\right) \omega, \frac{i}{2}\left(1+g^{2}\right) \omega, g \omega\right)
$$

Without loss of generality, we can assume that $\Sigma=\mathbb{C} \cup\{\infty\} \backslash\left\{p_{1}, \cdots, p_{n}\right\}$, where $p_{1}, \cdots, p_{n}$ are complex numbers and $g \neq 0, \infty$ at $p_{1}, \cdots, p_{n}$ (by rotating $M_{0}$ if necessary). Then $\omega$ needs a double pole at $p_{1}, \cdots, p_{n}$ so has $2 n-2$ zeros, counting multiplicity. Since $\omega$ needs a zero at each pole of $g$, with twice the multiplicity, it follows that $g$ has $n-1$ poles so has degree $n-1$. Hence we may write

$$
g=\frac{A(z)}{B(z)} \quad \text { and } \quad \omega=\frac{B(z)^{2} d z}{\prod_{i=1}^{n}\left(z-p_{i}\right)^{2}}
$$


where

$$
A(z)=\sum_{i=1}^{n} a_{i} z^{n-i} \quad \text { and } \quad B(z)=\sum_{i=1}^{n} b_{i} z^{n-i} .
$$

We are going to deform this Weierstrass data, so we see $a_{i}, b_{i}$ and $p_{i}$ for $1 \leq i \leq n$ as complex parameters. We denote by $\mathbf{x} \in \mathbb{C}^{3 n}$ the vector of these parameters, and by $\mathbf{x}_{0}$ the value of the parameters corresponding to the minimal $n$-noid $M_{0}$.

Let $\gamma_{i}$ be the homology class of a small circle centered at $p_{i}$ and define the following periods for $1 \leq i \leq n$ and $0 \leq k \leq 2$, depending on the parameter vector $\mathbf{x} \in \mathbb{C}^{3 n}$ :

Then

$$
\begin{gathered}
P_{i, k}(\mathbf{x})=\int_{\gamma_{i}} g^{k} \omega \\
P_{i}(\mathbf{x})=\left(P_{i, 0}(\mathbf{x}), P_{i, 1}(\mathbf{x}), P_{i, 2}(\mathbf{x})\right) \in \mathbb{C}^{3} \\
Q_{i}(\mathbf{x})=\int_{\gamma_{i}}\left(\frac{1}{2}\left(1-g^{2}\right) \omega, \frac{\mathrm{i}}{2}\left(1+g^{2}\right) \omega, g \omega\right) \in \mathbb{C}^{3} .
\end{gathered}
$$

$$
Q_{i}(\mathbf{x})=\left(\frac{1}{2}\left(P_{i, 0(\mathbf{x})}-P_{i, 2}(\mathbf{x})\right), \frac{\mathrm{i}}{2}\left(P_{i, 0}(\mathbf{x})+P_{i, 2}(\mathbf{x})\right), P_{i, 1}(\mathbf{x})\right) .
$$

The components of $Q_{i}\left(\mathbf{x}_{0}\right)$ are imaginary because the Period Problem is solved for $M_{0}$. This gives

$$
P_{i, 2}\left(\mathbf{x}_{0}\right)=\overline{P_{i, 0}\left(\mathbf{x}_{0}\right)} \quad \text { and } \quad P_{i, 1}\left(\mathbf{x}_{0}\right) \in \mathrm{i} \mathbb{R}
$$

Moreover, $\operatorname{Im}\left(Q_{i}\left(\mathbf{x}_{0}\right)\right)=-\phi_{i}$ where $\phi_{i}$ is the flux vector of $M_{0}$ at the end $p_{i}$. By the Residue Theorem, we have for all $\mathbf{x}$ in a neighborhood of $\mathbf{x}_{0}$ :

$$
\sum_{i=1}^{n} P_{i}(\mathbf{x})=0
$$

Let $P=\left(P_{1}, \cdots, P_{n-1}\right)$ and $Q=\left(Q_{1}, \cdots, Q_{n-1}\right)$.

Definition 1. $M_{0}$ is non-degenerate if the differential of $P$ (or equivalently, $Q$ ) at $\mathbf{x}_{0}$ has (complex) rank $3 n-3$.

Remark 1. If $n \geq 3$, we may (using Möbius transformations of the sphere) fix the value of three points, say $p_{1}, p_{2}, p_{3}$. Then "non-degenerate" means that the differential of $P$ with respect to the remaining parameters is an isomorphism of $\mathbb{C}^{3 n-3}$.

This notion is related to another standard notion of non-degeneracy:

Definition 2. $M_{0}$ is non-degenerate if its space of bounded Jacobi fields has (real) dimension 3.

Theorem 2. If $M_{0}$ is non-degenerate in the sense of Definition 2, then $M_{0}$ is non-degenerate in the sense of Definition 1 .

Proof. Assume $M_{0}$ is non-degenerate in the sense of Definition 2 . Then in a neighborhood of $M_{0}$, the space $\mathcal{M}$ of minimal $n$-noids (up to translation) is a smooth manifold of dimension $3 n-3$ by a standard application of the Implicit Function Theorem. Moreover, if we write $\phi_{i} \in \mathbb{R}^{3}$ for the flux vector at the $i$-th end, then the map $\phi=\left(\phi_{1}, \cdots, \phi_{n}\right)$ provides a local diffeomorphism between $\mathcal{M}$ and the space $V$ of vectors $v=\left(v_{1}, \cdots, v_{n}\right) \in\left(\mathbb{R}^{3}\right)^{n}$ such that $\sum_{i=1}^{n} v_{i}=0$. (All this is proved in Section 4 of [2] in the case where all ends are coplanar. The argument goes through 
in the general case.) Hence given a vector $v \in V$, there exists a deformation $M_{t}$ of $M_{0}$ such that $M_{t} \in \mathcal{M}$ and $\left.\frac{d}{d t} \phi\left(M_{t}\right)\right|_{t=0}=v$. We may write the Weierstrass data of $M_{t}$ as above and obtain a set of parameters $\mathbf{x}(t)$, depending smoothly on $t$, such that $\mathbf{x}(0)=\mathbf{x}_{0}$. Then $d Q\left(\mathbf{x}_{0}\right) \cdot \mathbf{x}^{\prime}(0)=-\mathbf{i} v$. Since $Q$ is holomorphic, its differential is complex-linear so $d Q\left(\mathbf{x}_{0}\right)$ has complex rank equal to $\operatorname{dim} V=3 n-3$.

If all ends of $M_{0}$ have coplanar axes, then $M_{0}$ is non-degenerate in the sense of Definition 2 by Proposition 2 in [2]. In particular, the (most symmetric) $n$-noids of Jorge-Meeks are nondegenerate. This implies that generic $n$-noids in the component of the Jorge-Meeks $n$-noid are non-degenerate.

\section{BACKGROUND}

In this section, we recall standard notations and results used in the DPW method. We work in the "untwisted" setting.

3.1. Loop groups. A loop is a smooth map from the unit circle $\mathbb{S}^{1}=\{\lambda \in \mathbb{C}:|\lambda|=1\}$ to a matrix group. The circle variable is denoted $\lambda$ and called the spectral parameter. For $\rho>0$, we denote $\mathbb{D}_{\rho}=\{\lambda \in \mathbb{C}:|\lambda|<\rho\}, \mathbb{D}_{\rho}^{*}=\mathbb{D}_{\rho} \backslash\{0\}$ and $\mathbb{D}=\mathbb{D}_{1}$.

- If $G$ is a matrix Lie group (or Lie algebra), $\Lambda G$ denotes the group (or algebra) of smooth maps $\Phi: \mathbb{S}^{1} \rightarrow G$.

- $\Lambda_{+} S L(2, \mathbb{C}) \subset \Lambda S L(2, \mathbb{C})$ is the subgroup of maps $B$ which extend holomorphically to $\mathbb{D}$ with $B(0)$ upper triangular.

- $\Lambda_{+}^{\mathbb{R}} S L(2, \mathbb{C}) \subset \Lambda_{+} S L(2, \mathbb{C})$ is the subgroup of maps $B$ such that $B(0)$ has positive entries on the diagonal.

Theorem 3 (Iwasawa decomposition). The multiplication $\Lambda S U(2) \times \Lambda_{+}^{\mathbb{R}} S L(2, \mathbb{C}) \rightarrow \Lambda S L(2, \mathbb{C})$ is a diffeomorphism. The unique splitting of an element $\Phi \in \Lambda S L(2, \mathbb{C})$ as $\Phi=F B$ with $F \in \Lambda S U(2)$ and $B \in \Lambda_{+}^{\mathbb{R}} S L(2, \mathbb{C})$ is called Iwasawa decomposition. $F$ is called the unitary factor of $\Phi$ and denoted $\operatorname{Uni}(\Phi) . B$ is called the positive factor and denoted $\operatorname{Pos}(\Phi)$.

3.2. The matrix model of $\mathbb{R}^{3}$. In the DPW method, one identifies $\mathbb{R}^{3}$ with the Lie algebra $\mathfrak{s u}(2)$ by

$$
x=\left(x_{1}, x_{2}, x_{3}\right) \in \mathbb{R}^{3} \longleftrightarrow X=-\mathrm{i}\left(\begin{array}{cc}
-x_{3} & x_{1}+\mathrm{i} x_{2} \\
x_{1}-\mathrm{i} x_{2} & x_{3}
\end{array}\right) \in \mathfrak{s u}(2) .
$$

We have $\operatorname{det}(X)=\|x\|^{2}$. The group $S U(2)$ acts as linear isometries on $\mathfrak{s u}(2)$ by conjugation: $H \cdot X=H X H^{-1}$.

3.3. The DPW method. The input data for the DPW method is a quadruple $\left(\Sigma, \xi, z_{0}, \phi_{0}\right)$ where:

- $\Sigma$ is a Riemann surface.

- $\xi=\xi(z, \lambda)$ is a $\Lambda \mathfrak{s l}(2, \mathbb{C})$-valued holomorphic 1 -form on $\Sigma$ called the DPW potential. More precisely,

$$
\xi=\left(\begin{array}{cc}
\alpha & \lambda^{-1} \beta \\
\gamma & -\alpha
\end{array}\right)
$$

where $\alpha(z, \lambda), \beta(z, \lambda), \gamma(z, \lambda)$ are holomorphic 1 -forms on $\Sigma$ with respect to the $z$ variable, and are holomorphic with respect to $\lambda$ in the disk $\mathbb{D}_{\rho}$ for some $\rho>1$. 
- $z_{0} \in \Sigma$ is a base point.

- $\phi_{0} \in \Lambda S L(2, \mathbb{C})$ is an initial condition.

Given this data, the DPW method is the following procedure.

- Let $\widetilde{\Sigma}$ be the universal cover of $\Sigma$ and $\widetilde{z}_{0} \in \widetilde{\Sigma}$ be an arbitrary element in the fiber of $z_{0}$. Solve the Cauchy Problem on $\widetilde{\Sigma}$ :

$$
\left\{\begin{array}{l}
d \Phi(z, \lambda)=\Phi(z, \lambda) \xi(z, \lambda) \\
\Phi\left(\widetilde{z}_{0}, \lambda\right)=\phi_{0}(\lambda)
\end{array}\right.
$$

to obtain a solution $\Phi: \widetilde{\Sigma} \rightarrow \Lambda S L(2, \mathbb{C})$.

- Compute the Iwasawa decomposition $(F(z, \cdot), B(z, \cdot))$ of $\Phi(z, \cdot)$.

- Define $f: \widetilde{\Sigma} \rightarrow \mathfrak{s u}(2) \sim \mathbb{R}^{3}$ by the Sym-Bobenko formula:

$$
f(z)=-2 \mathrm{i} \frac{\partial F}{\partial \lambda}(z, 1) F(z, 1)^{-1}=: \operatorname{Sym}(F(z, \cdot)) .
$$

Then $f$ is a CMC-1 (branched) conformal immersion. $f$ is regular at $z$ (meaning unbranched) if and only if $\beta(z, 0) \neq 0$. Its Gauss map is given by

$$
N(z)=-\mathrm{i} F(z, 1)\left(\begin{array}{cc}
-1 & 0 \\
0 & 1
\end{array}\right) F(z, 1)^{-1}=: \operatorname{Nor}(F(z, \cdot)) .
$$

The differential of $f$ satisfies

$$
d f(z)=2 \mathrm{i} B_{11}(z, 0)^{2} F(z, 1)\left(\begin{array}{cc}
0 & \beta(z, 0) \\
\bar{\beta}(z, 0) & 0
\end{array}\right) F(z, 1)^{-1} .
$$

Remark 2. (1) In [14, I have opposite signs in Equations (6) and (7). This is unfortunate because it makes the basis $\left(f_{x}, f_{y}, N\right)$ negatively oriented. Equation (6) is the right formula, which one obtains by untwisting the standard Sym-Bobenko formula in the twisted case. See [9] or [13].

(2) I have not been able to find Formula (8) in the litterature. Of course, the DPW method constructs a moving frame for $f$, so one has a formula for $d f$, but usually it is written in a special coordinate system and only in the "twisted" setting. For the interested reader, I derive Equation (8) from the Sym-Bobenko formula at the end of Appendix A.

3.4. The Monodromy Problem. Assume that $\Sigma$ is not simply connected so its universal cover $\widetilde{\Sigma}$ is not trivial. Let $\operatorname{Deck}(\widetilde{\Sigma} / \Sigma)$ be the group of fiber-preserving diffeomorphisms of $\widetilde{\Sigma}$. For $\gamma \in \operatorname{Deck}(\widetilde{\Sigma} / \Sigma)$, let

$$
\mathcal{M}_{\gamma}(\Phi)(\lambda)=\Phi(\gamma(z), \lambda) \Phi(z, \lambda)^{-1}
$$

be the monodromy of $\Phi$ with respect to $\gamma$ (which is independent of $z \in \widetilde{\Sigma}$ ). The standard condition which ensures that the immersion $f$ descends to a well defined immersion on $\Sigma$ is the following system of equations, called the Monodromy Problem.

$$
\forall \gamma \in \operatorname{Deck}(\widetilde{\Sigma} / \Sigma) \quad\left\{\begin{array}{l}
\mathcal{M}_{\gamma}(\Phi) \in \Lambda S U(2) \\
\mathcal{M}_{\gamma}(\Phi)(1)= \pm I_{2} \\
\frac{\partial \mathcal{M}_{\gamma}(\Phi)}{\partial \lambda}(1)=0
\end{array}\right.
$$


One can identify $\operatorname{Deck}(\widetilde{\Sigma} / \Sigma)$ with the fundamental group $\pi_{1}\left(\Sigma, z_{0}\right)$ (see for example Theorem 5.6 in [4), so we will in general see $\gamma$ as an element of $\pi_{1}\left(\Sigma, z_{0}\right)$. Under this identification, the monodromy of $\Phi$ with respect to $\gamma \in \pi_{1}\left(\Sigma, z_{0}\right)$ is given by

$$
\mathcal{M}_{\gamma}(\Phi)(\lambda)=\Phi(\widetilde{\gamma}(1), \lambda) \Phi(\widetilde{\gamma}(0), \lambda)^{-1}
$$

where $\widetilde{\gamma}:[0,1] \rightarrow \widetilde{\Sigma}$ is the lift of $\gamma$ such that $\widetilde{\gamma}(0)=\widetilde{z}_{0}$.

\subsection{Gauging.}

Definition 3. A gauge on $\Sigma$ is a map $G: \Sigma \rightarrow \Lambda_{+} S L(2, \mathbb{C})$ such that $G(z, \lambda)$ depends holomorphically on $z \in \Sigma$ and $\lambda \in \mathbb{D}_{\rho}$ for some $\rho>1$.

Let $\Phi$ be a solution of $d \Phi=\Phi \xi$ and $G$ be a gauge. Let $\widehat{\Phi}=\Phi \times G$. Then $\widehat{\Phi}$ and $\Phi$ define the same immersion $f$. This is called "gauging". The gauged potential is

$$
\widehat{\xi}=\widehat{\Phi}^{-1} d \widehat{\Phi}=G^{-1} \xi G+G^{-1} d G
$$

and will be denoted $\xi \cdot G$, the dot denoting the action of the gauge group on the potential.

3.6. Functional spaces. We decompose a smooth function $f: \mathbb{S}^{1} \rightarrow \mathbb{C}$ in Fourier series

$$
f(\lambda)=\sum_{i \in \mathbb{Z}} f_{i} \lambda^{i}
$$

Fix some $\rho>1$ and define

$$
\|f\|=\sum_{i \in \mathbb{Z}}\left|f_{i}\right| \rho^{|i|}
$$

Let $\mathcal{W}_{\rho}$ be the space of functions $f$ with finite norm. This is a Banach algebra, classically called the Wiener algebra when $\rho=1$ (see Proposition 7 in appendix A). Functions in $\mathcal{W}_{\rho}$ extend holomorphically to the annulus $\frac{1}{\rho}<|\lambda|<\rho$.

We define $\mathcal{W}_{\bar{\rho}}^{\geq 0}, \mathcal{W}_{\rho}^{>0}, \mathcal{W}_{\bar{\rho}}^{\leq 0}$ and $\mathcal{W}_{\rho}^{<0}$ as the subspaces of functions $f$ such that $f_{i}=0$ for $i<0, i \leq 0, i>0$ and $i \geq 0$, respectively. Functions in $\mathcal{W}_{\bar{\rho}}^{\geq 0}$ extend holomorphically to the disk $\mathbb{D}_{\rho}$ and satisfy $|f(\lambda)| \leq\|f\|$ for all $\lambda \in \mathbb{D}_{\rho}$. We write $\mathcal{W}^{0} \sim \mathbb{C}$ for the subspace of constant functions, so we have a direct sum $\mathcal{W}_{\rho}=\mathcal{W}_{\rho}^{<0} \oplus \mathcal{W}^{0} \oplus \mathcal{W}_{\rho}^{>0}$. A function $f$ will be decomposed as $f=f^{-}+f^{0}+f^{+}$with $\left(f^{-}, f^{0}, f^{+}\right) \in \mathcal{W}_{\rho}^{<0} \times \mathcal{W}^{0} \times \mathcal{W}_{\rho}^{>0}$.

We define the star operator by

$$
f^{*}(\lambda)=\overline{f\left(\frac{1}{\bar{\lambda}}\right)}=\sum_{i \in \mathbb{Z}} \overline{f_{-i}} \lambda^{i}
$$

The involution $f \mapsto f^{*}$ exchanges $\mathcal{W}_{\bar{\rho}}^{\geq 0}$ and $\mathcal{W}_{\bar{\rho}}^{\leq 0}$. We have $\lambda^{*}=\lambda^{-1}$ and $c^{*}=\bar{c}$ if $c$ is a constant. A function $f$ is real on the unit circle if and only if $f=f^{*}$.

If $L$ is a loop group, we denote $L_{\rho} \subset L$ the subgroup of loops whose entries are in $\mathcal{W}_{\rho}$. If $\Phi \in \Lambda S L(2, \mathbb{C})_{\rho}$ and $(F, B)$ is its Iwasawa decomposition, then in fact $F \in \Lambda S U(2)_{\rho}$ and $B \in \Lambda_{+}^{\mathbb{R}} S L(2, \mathbb{C})_{\rho}$ (see Proposition 8 in Appendix A). Moreover, Iwasawa decomposition is smooth, as a map between Banach manifolds (see Theorem 5 in Appendix A). 


\section{A BLOW-UP RESULT}

In this section, we consider a one-parameter family of DPW potential $\xi_{t}$ with solution $\Phi_{t}$ and assume that $\Phi_{0}(z, \lambda)$ is independent of $\lambda$. Then its unitary part $F_{0}(z, \lambda)$ is independent of $\lambda$. The Sym Bobenko formula yields that $f_{0} \equiv 0$, so the family $f_{t}$ collapses to the origin as $t=0$. The following theorem says that the blow-up $\frac{1}{t} f_{t}$ converges to a minimal surface whose Weierstrass data is explicitly computed.

Theorem 4. Let $\Sigma$ be a Riemann surface, $\left(\xi_{t}\right)_{t \in I}$ a family of DPW potentials on $\Sigma$ and $\left(\Phi_{t}\right)_{t \in I}$ a family of solutions of $d \Phi_{t}=\Phi_{t} \xi_{t}$ on the universal cover $\widetilde{\Sigma}$ of $\Sigma$, where $I \subset \mathbb{R}$ is a neighborhood of 0 . Fix a base point $z_{0} \in \widetilde{\Sigma}$. Assume that

(1) $(t, z) \mapsto \xi_{t}(z, \cdot)$ and $t \mapsto \Phi_{t}\left(z_{0}, \cdot\right)$ are $C^{1}$ maps into $\Lambda \mathfrak{s l}(2, \mathbb{C})_{\rho}$ and $\Lambda S L(2, \mathbb{C})_{\rho}$, respectively.

(2) For all $t \in I, \Phi_{t}$ solves the Monodromy Problem (9).

(3) $\Phi_{0}(z, \lambda)$ is independent of $\lambda$ :

$$
\Phi_{0}(z, \lambda)=\left(\begin{array}{ll}
a(z) & b(z) \\
c(z) & d(z)
\end{array}\right)
$$

Let $f_{t}=\operatorname{Sym}\left(\operatorname{Uni}\left(\Phi_{t}\right)\right): \Sigma \rightarrow \mathbb{R}^{3}$ be the CMC-1 immersion given by the DPW method. Then

$$
\lim _{t \rightarrow 0} \frac{1}{t} f_{t}(z)=\psi(z)
$$

where $\psi: \Sigma \rightarrow \mathbb{R}^{3}$ is a (possibly branched) minimal immersion with the following Weierstrass data:

$$
g(z)=\frac{-a(z)}{c(z)} \quad \text { and } \quad \omega=\left.4 c(z)^{2} \frac{\partial \xi_{t ; 12}^{(-1)}}{\partial t}\right|_{t=0} .
$$

The limit is for the uniform $C^{1}$ convergence on compact subsets of $\Sigma$.

Here $\xi_{t ; 12}^{(-1)}$ denotes the coefficient of $\lambda^{-1}$ in the upper right entry of $\xi_{t}$. In case $\omega=0$, the minimal immersion degenerates into a point and $\psi$ is constant.

Proof: by standard ODE theory, $(t, z) \mapsto \Phi_{t}(z, \cdot)$ is a $C^{1}$ map into $\Lambda S L(2, \mathbb{C})_{\rho}$. Let $\left(F_{t}, B_{t}\right)$ be the Iwasawa decomposition of $\Phi_{t}$. By Theorem 5 in Appendix A $(t, z) \mapsto F_{t}(z, \cdot)$ and $(t, z) \mapsto B_{t}(z, \cdot)$ are $C^{1}$ maps into $\Lambda S U(2)_{\rho}$ and $\Lambda_{+}^{\mathbb{R}} S L(2, \mathbb{C})_{\rho}$, respectively. At $t=0$, $\Phi_{0}$ is constant with respect to $\lambda$, so its Iwasawa decomposition is the standard $Q R$ decomposition:

$$
F_{0}=\frac{1}{\sqrt{|a|^{2}+|c|^{2}}}\left(\begin{array}{cc}
a & -\bar{c} \\
c & \bar{a}
\end{array}\right) \quad B_{0}=\frac{1}{\sqrt{|a|^{2}+|c|^{2}}}\left(\begin{array}{cc}
|a|^{2}+|c|^{2} & \bar{a} b+\bar{c} d \\
0 & 1
\end{array}\right) .
$$

The Sym-Bobenko formula (6) yields $f_{0}=0$. Let $\mu_{t}=B_{t ; 11}^{0}$ and $\beta_{t}=\xi_{t ; 12}^{(-1)}$. By Equation (8), we have

$$
d f_{t}(z)=2 \mathrm{i} \mu_{t}(z)^{2} F_{t}(z, 1)\left(\begin{array}{cc}
0 & \beta_{t}(z) \\
\bar{\beta}_{t}(z) & 0
\end{array}\right) F_{t}(z, 1)^{-1}
$$


Hence $(t, z) \mapsto d f_{t}(z)$ is a $C^{1}$ map. At $t=0, \xi_{0}$ is constant with respect to $\lambda$, so $\beta_{0}=0$. Define $\widetilde{f}_{t}(z)=\frac{1}{t} f_{t}(z)$ for $t \neq 0$. Then $d \widetilde{f}_{t}(z)$ extends at $t=0$, as a continous function of $(t, z)$, by

$$
\begin{aligned}
d \widetilde{f}_{0}=\left.\frac{d}{d t} d f_{t}\right|_{t=0} & =2 \mathrm{i}\left(\begin{array}{cc}
a & -\bar{c} \\
c & \bar{a}
\end{array}\right)\left(\begin{array}{cc}
0 & \beta^{\prime} \\
\overline{\beta^{\prime}} & 0
\end{array}\right)\left(\begin{array}{cc}
\bar{a} & \bar{c} \\
-c & a
\end{array}\right) \\
& =2 \mathrm{i}\left(\begin{array}{cc}
-a c \beta^{\prime}-\overline{a c \beta^{\prime}} & a^{2} \beta^{\prime}-\overline{c^{2} \beta^{\prime}} \\
\overline{a^{2} \beta^{\prime}}-c^{2} \beta^{\prime} & a c \beta^{\prime}+\overline{a c \beta^{\prime}}
\end{array}\right)
\end{aligned}
$$

where $\beta^{\prime}=\left.\frac{d}{d t} \beta_{t}\right|_{t=0}$. In euclidean coordinates, this gives

$$
d \widetilde{f}_{0}=4 \operatorname{Re}\left[\frac{1}{2}\left(c^{2}-a^{2}\right) \beta^{\prime}, \frac{i}{2}\left(c^{2}+a^{2}\right) \beta^{\prime},-a c \beta^{\prime}\right] .
$$

Writing $g=\frac{-a}{c}$ and $\omega=4 c^{2} \beta^{\prime}$, we obtain

$$
\widetilde{f}_{0}(z)=\widetilde{f}_{0}\left(z_{0}\right)+\operatorname{Re} \int_{z_{0}}^{z}\left[\frac{1}{2}\left(1-g^{2}\right) \omega, \frac{\dot{1}}{2}\left(1+g^{2}\right) \omega, g \omega\right]
$$

and we see that $\widetilde{f}_{0}$ is a minimal surface with Weierstrass data $(g, \omega)$. The last statement of Theorem 4 comes from the fact that $d \widetilde{f}_{t}$ converges uniformly to $d \widetilde{f}_{0}$ on compact subsets of $\Sigma$.

4.1. Example. As an example, we consider the family of Delaunay surfaces given by the following DPW potential in $\mathbb{C}^{*}$ :

$$
\xi_{t}(z, \lambda)=\left(\begin{array}{cc}
0 & \lambda^{-1} r+s \\
\lambda r+s & 0
\end{array}\right) \frac{d z}{z} \quad \text { with } \quad\left\{\begin{array}{l}
r+s=\frac{1}{2} \\
r s=t \\
r<s
\end{array}\right.
$$

with initial condition $\Phi_{t}(1)=I_{2}$. As $t \rightarrow 0$, we have $(r, s) \rightarrow\left(0, \frac{1}{2}\right)$. We have

$$
\begin{gathered}
\Phi_{0}(z, \lambda)=\exp \left(\begin{array}{cc}
0 & \frac{1}{2} \\
\frac{1}{2} & 0
\end{array}\right) \log z=\frac{1}{2 \sqrt{z}}\left(\begin{array}{cc}
z+1 & z-1 \\
z-1 & z+1
\end{array}\right) \\
\left.\frac{\partial \xi_{t}}{\partial t}\right|_{t=0}=\left(\begin{array}{cc}
0 & 2 \lambda^{-1} \\
2 \lambda & 0
\end{array}\right) \frac{d z}{z} .
\end{gathered}
$$

Theorem 4 applies and gives

$$
g(z)=\frac{1+z}{1-z} \quad \text { and } \quad \omega(z)=4\left(\frac{z-1}{2 \sqrt{z}}\right)^{2} \frac{2 d z}{z}=2\left(\frac{z-1}{z}\right)^{2} d z .
$$

This is the Weierstrass data of a horizontal catenoid of waist-radius 4 and axis $O x_{1}$, with $x_{1} \rightarrow$ $+\infty$ at the end $z=0$.

\section{The DPW Potential}

We now start the proof of Theorem 1. Let $(g, \omega)$ be the Weierstrass data of the given minimal $n$-noid $M_{0}$, written as in Section 2. We introduce $3 n \lambda$-dependent parameters $a_{i}, b_{i}$ and $p_{i}$ for $1 \leq i \leq n$ in the functional space $\mathcal{W}_{\bar{\rho}}^{\geq 0}$. The vector of these parameters is denoted $\mathbf{x} \in\left(\mathcal{W}_{\bar{\rho}}^{\geq 0}\right)^{3 n}$. The parameter $\mathbf{x}$ is in a neighborhood of a (constant) central value $\mathbf{x}_{0} \in\left(\mathcal{W}^{0}\right)^{3 n}$ which correspond to the Weierstrass data of $M_{0}$, written as in Section 2. We define

$$
A_{\mathbf{x}}(z, \lambda)=\sum_{i=1}^{n} a_{i}(\lambda) z^{n-i}
$$




$$
\begin{gathered}
B_{\mathbf{x}}(z, \lambda)=\sum_{i=1}^{n} b_{i}(\lambda) z^{n-i} \\
g_{\mathbf{x}}(z, \lambda)=\frac{A_{\mathbf{x}}(z, \lambda)}{B_{\mathbf{x}}(z, \lambda)} \\
\omega_{\mathbf{x}}(z, \lambda)=\frac{B_{\mathbf{x}}(z, \lambda)^{2} d z}{\prod_{i=1}^{n}\left(z-p_{i}(\lambda)\right)^{2}} .
\end{gathered}
$$

For $t$ in a neighborhood of 0 in $\mathbb{R}$, we consider the following DPW potential:

$$
\xi_{t, \mathbf{x}}(z, \lambda)=\left(\begin{array}{cc}
0 & \frac{1}{4} t(\lambda-1)^{2} \lambda^{-1} \omega_{\mathbf{x}}(z, \lambda) \\
d g_{\mathbf{x}}(z, \lambda) & 0
\end{array}\right)
$$

We fix a base point $z_{0}$, away from the poles of $g$ and $\omega$, and we take the initial condition

$$
\phi_{0}(\lambda)=\left(\begin{array}{cc}
g_{\mathbf{x}}\left(z_{0}, \lambda\right) & 1 \\
-1 & 0
\end{array}\right)
$$

These choices are motivated by the following observations:

(1) At $t=0$, we have

$$
\xi_{0, \mathbf{x}}(z, \lambda)=\left(\begin{array}{cc}
0 & 0 \\
d g_{\mathbf{x}}(z, \lambda) & 0
\end{array}\right)
$$

The solution of the Cauchy Problem (5) is given by

$$
\Phi_{0, \mathbf{x}}(z, \lambda)=\left(\begin{array}{cc}
g_{\mathbf{x}}(z, \lambda) & 1 \\
-1 & 0
\end{array}\right)
$$

which is well-defined, so the Monodromy Problem (9) is solved at $t=0$.

(2) The same conclusion holds if $\lambda=1$ instead of $t=0$. In particular, Items (ii) and (iii) of the Monodromy Problem (9) are automatically solved.

(3) At $\mathbf{x}=\mathbf{x}_{0}$, we have $g_{\mathbf{x}_{0}}=g$ so $\Phi_{0, \mathbf{x}_{0}}(z, \lambda)$ is independent of $\lambda$. Moreover,

$$
\left.\frac{\partial \xi_{t, \mathbf{x}_{0} ; 12}^{(-1)}}{\partial t}\right|_{t=0}=\frac{\omega}{4} .
$$

Provided the Monodromy Problem is solved for all $t$ in a neighborhood of 0 , Theorem 4 applies and the limit minimal surface has Weierstrass data $(g, \omega)$ so is the minimal $n$-noid $M_{0}$, up to translation (see details in Section 7.1).

5.1. Regularity. Our potential $\xi_{t, \mathbf{x}}$ has poles at the zeros of $B_{\mathbf{x}}$ and the points $p_{1}, \cdots, p_{n}$. (At $\infty$, we have $\omega_{\mathbf{x}} \sim b_{1}^{2} z^{-2} d z$ which is holomorphic.) We want the zeros of $B_{\mathbf{x}}$ to be apparent singularities, so we require the potential to be gauge-equivalent to a regular potential in a neighborhood of these points. Consider the gauge

$$
G_{\mathbf{x}}(z, \lambda)=\left(\begin{array}{cc}
g_{\mathbf{x}}(z, \lambda)^{-1} & -1 \\
0 & g_{\mathbf{x}}(z, \lambda)
\end{array}\right)
$$

The gauged potential is

$$
\widehat{\xi}_{t, \mathbf{x}}:=\xi_{t, \mathbf{x}} \cdot G_{\mathbf{x}}=\left(\begin{array}{cc}
0 & \frac{1}{4} t(\lambda-1)^{2} \lambda^{-1} g_{\mathbf{x}}^{2} \omega_{\mathbf{x}} \\
g_{\mathbf{x}}^{-2} d g_{\mathbf{x}} & 0
\end{array}\right)
$$


We have

$$
g_{\mathbf{x}}^{-2} d g_{\mathbf{x}}=\frac{A_{\mathbf{x}}^{\prime} B_{\mathbf{x}}-A_{\mathbf{x}} B_{\mathbf{x}}^{\prime}}{A_{\mathbf{x}}^{2}} \quad \text { and } \quad g_{\mathbf{x}}^{2} \omega_{\mathbf{x}}=\frac{A_{\mathbf{x}}^{2} d z}{\prod_{i=1}^{n}\left(z-p_{i}\right)^{2}} .
$$

Let $\zeta$ be a zero of $B_{\mathbf{x}_{0}}$ (recall that $B_{\mathbf{x}_{0}}$ does not depend on $\lambda$ ). Then $A_{\mathbf{x}_{0}}(\zeta) \neq 0$. By continuity, there exists a neighborhood $U$ of $\zeta$ such that for $z \in U, \lambda \in \mathbb{D}_{\rho}$ and $\mathbf{x}$ close enough to $\mathbf{x}_{0}$, $A_{\mathbf{x}}(z, \lambda) \neq 0$. So $\widehat{\xi}_{t, \mathbf{x}}$ is holomorphic in $U \times \mathbb{D}_{\rho}^{*}$ and moreover, $\widehat{\xi}_{t, \mathbf{x} ; 12}^{(-1)} \neq 0$. This ensures that the immersion extends analytically to $U$ and is unbranched in $U$.

\section{The MONODROMY PROBLEM}

6.1. Formulation of the problem. For $i \in[1, n]$, we denote $p_{i, 0}$ the central value of the parameter $p_{i}$ (so $p_{1,0}, \cdots, p_{n, 0}$ are the ends of the minimal $n$-noid $M_{0}$ ). We consider the following $\lambda$-independent domain on the Riemann sphere:

$$
\Omega=\left\{z \in \mathbb{C}: \forall i \in[1, n],\left|z-p_{i, 0}\right|>\varepsilon\right\} \cup\{\infty\}
$$

where $\varepsilon>0$ is a fixed, small enough number such that the disks $D\left(p_{i, 0}, 8 \varepsilon\right)$ for $1 \leq i \leq n$ are disjoint. As in [14], we first construct a family of immersions $f_{t}$ on $\Omega$. Then we extend $f_{t}$ to an $n$-punctured sphere in Proposition 3.

Let $\widetilde{\Omega}$ be the universal cover of $\Omega$ and $\Phi_{t, \mathbf{x}}(z, \lambda)$ be the solution of the following Cauchy Problem on $\widetilde{\Omega}$ :

$$
\left\{\begin{array}{l}
d \Phi_{t, \mathbf{x}}(z, \lambda)=\Phi_{t, \mathbf{x}}(z, \lambda) \xi_{t, \mathbf{x}}(z, \lambda) \\
\Phi_{t, \mathbf{x}}\left(\widetilde{z}_{0}, \lambda\right)=\phi_{0}
\end{array}\right.
$$

We denote $\gamma_{1}, \cdots, \gamma_{n-1}$ a set of generators of the fundamental group $\pi_{1}\left(\Omega, z_{0}\right)$, with $\gamma_{i}$ encircling the point $p_{i, 0}$. We may assume that each $\gamma_{i}$ is represented by a fixed curve avoiding the poles of $\xi_{t, \mathbf{x}}$. Let

$$
M_{i}(t, \mathbf{x})=\mathcal{M}_{\gamma_{i}}\left(\Phi_{t, \mathbf{x}}\right)
$$

be the monodromy of $\Phi_{t, \mathbf{x}}$ along $\gamma_{i}$. By Equation $(12)$, we have $M_{i}(0, \mathbf{x})=I_{2}$. Recall that the matrix exponential is a local diffeomorphism from a neighborhood of 0 in the Lie algebra $\mathfrak{s l}(2, \mathbb{C})$ (respectively $\mathfrak{s u}(2)$ ) to a neighborhood of $I_{2}$ in $S L(2, \mathbb{C})$ (respectively $S U(2)$ ). The inverse diffeomorphism is denoted log. For $t \neq 0$ small enough and $\lambda \in \mathbb{D}_{\rho} \backslash\{1\}$, we define as in [14]

$$
\widetilde{M}_{i}(t, \mathbf{x})(\lambda)=\frac{4 \lambda}{t(\lambda-1)^{2}} \log M_{i}(t, \mathbf{x})(\lambda) .
$$

Proposition 1. (1) $\widetilde{M}_{i}(t, \mathbf{x})(\lambda)$ extends smoothly at $t=0$ and $\lambda=1$, and each entry $\widetilde{M}_{i ; k \ell}$ is a smooth map from a neighborhood of $\left(0, \mathbf{x}_{0}\right)$ in $\mathbb{R} \times\left(\mathcal{W}_{\bar{\rho}}^{\geq 0}\right)^{3}$ to $\mathcal{W}_{\rho}$.

(2) At $t=0$, we have

$$
\widetilde{M}_{i}(0, \mathbf{x})(\lambda)=\left(\begin{array}{cc}
\mathcal{P}_{i, 1}(\mathbf{x}) & \mathcal{P}_{i, 2}(\mathbf{x}) \\
-\mathcal{P}_{i, 0}(\mathbf{x}) & -\mathcal{P}_{i, 1}(\mathbf{x})
\end{array}\right) \quad \text { where } \mathcal{P}_{i, k}(\mathbf{x})=\int_{\gamma_{i}} g_{\mathbf{x}}^{k} \omega_{\mathbf{x}}
$$

(3) The Monodromy Problem (9) is equivalent to

$$
\widetilde{M}_{i}(t, \mathbf{x}) \in \Lambda \mathfrak{s u}(2) \quad \text { for } \quad 1 \leq i \leq n-1 .
$$


Proof: we follow the proof of Proposition 1 in [14]. We first consider the case where the parameter $\mathbf{x}=\left(a_{i}, b_{i}, p_{i}\right)_{1 \leq i \leq n}$ is constant with respect to $\lambda$, so $\mathbf{x} \in \mathbb{C}^{3 n}$. For $(\mu, \mathbf{x})$ in a neighborhood of $\left(0, \mathbf{x}_{0}\right)$ in $\overline{\mathbb{C}} \times \mathbb{C}^{3 n}$, we define

$$
\widehat{\xi}_{\mu, \mathbf{x}}(z)=\left(\begin{array}{cc}
0 & \mu \omega_{\mathbf{x}}(z) \\
d g_{\mathbf{x}}(z) & 0
\end{array}\right)
$$

where $\omega_{\mathbf{x}}$ and $g_{\mathbf{x}}$ are defined by Equations (10) and (11), except that $a_{i}, b_{i}, p_{i}$ are constant complex numbers. Let $\widehat{\Phi}_{\mu, \mathbf{x}}$ be the solution of the Cauchy Problem $d \widehat{\Phi}_{\mu, \mathbf{x}}=\widehat{\Phi}_{\mu, \mathbf{x}} \widehat{\xi}_{\mu, \mathbf{x}}$ in $\widetilde{\Omega}$ with initial condition $\widehat{\Phi}_{\mu, \mathbf{x}}\left(\widetilde{z}_{0}\right)=\phi_{0}$. Let $N_{i}(\mu, \mathbf{x})=\mathcal{M}_{\gamma_{i}}\left(\widehat{\Phi}_{\mu, \mathbf{x}}\right)$. By standard ODE theory, each entry of $N_{i}$ is a holomorphic function of $(\mu, \mathbf{x})$. At $\mu=0, \widehat{\Phi}_{0, \mathbf{x}}$ is given by Equation (12), so in particular $N_{i}(0, \mathbf{x})=I_{2}$. Hence

$$
\widetilde{N}_{i}(\mu, \mathbf{x}):=\frac{1}{\mu} \log N_{i}(\mu, \mathbf{x})
$$

extends holomorphically at $\mu=0$ with value $\widetilde{N}_{i}(0, \mathbf{x})=\frac{\partial N_{i}}{\partial \mu}(0, \mathbf{x})$. By Proposition 8 in Appendix A of [14],

$$
\frac{\partial N_{i}}{\partial \mu}(0, \mathbf{x})=\left.\int_{\gamma_{i}} \widehat{\Phi}_{0, \mathbf{x}} \frac{\partial \widehat{\xi}_{\mu, \mathbf{x}}}{\partial \mu}\right|_{\mu=0} \widehat{\Phi}_{0, \mathbf{x}}^{-1}
$$

Hence

$$
\widetilde{N}_{i}(0, \mathbf{x})=\int_{\gamma_{i}}\left(\begin{array}{cc}
g_{\mathbf{x}} & 1 \\
-1 & 0
\end{array}\right)\left(\begin{array}{cc}
0 & \omega_{\mathbf{x}} \\
0 & 0
\end{array}\right)\left(\begin{array}{cc}
0 & -1 \\
1 & g_{\mathbf{x}}
\end{array}\right)=\int_{\gamma_{i}}\left(\begin{array}{cc}
g_{\mathbf{x}} \omega_{\mathbf{x}} & g_{\mathbf{x}}^{2} \omega_{\mathbf{x}} \\
-\omega_{\mathbf{x}} & -g_{\mathbf{x}} \omega_{\mathbf{x}}
\end{array}\right) .
$$

For $(t, \mathbf{x})$ in a neighborhood of $\left(0, \mathbf{x}_{0}\right)$ in $\mathbb{R} \times\left(\mathcal{W}_{\bar{\rho}}^{\geq 0}\right)^{3 n}$, we have

$$
\xi_{t, \mathbf{x}}(z, \lambda)=\widehat{\xi}_{\mu(t, \lambda), \mathbf{x}(\lambda)}(z) \quad \text { with } \quad \mu(t, \lambda)=\frac{t(\lambda-1)^{2}}{4 \lambda}
$$

Hence

$$
M_{i}(t, \mathbf{x})(\lambda)=N_{i}(\mu(t, \lambda), \mathbf{x}(\lambda)) \quad \text { and } \quad \widetilde{M}_{i}(t, \mathbf{x})(\lambda)=\widetilde{N}_{i}(\mu(t, \lambda), \mathbf{x}(\lambda)) .
$$

By substitution (see Proposition 9 in Appendix B of [14]), each entry of $\widetilde{M}_{i}$ is is a smooth map from a neighborhood of $\left(0, \mathbf{x}_{0}\right)$ in $\mathbb{R} \times\left(\mathcal{W}_{\bar{\rho}}^{\geq 0}\right)^{3}$ to $\mathcal{W}_{\rho}$. Moreover, $\widetilde{M}_{i}(0, \mathbf{x})$ is given by Equation 17). The fact that $\widetilde{M}_{i}$ extends holomorphically at $\lambda=1$ implies that Points (ii) and (iii) of Problem (9) are automatically satisfied. Since $\lambda^{-1}(\lambda-1)^{2} \in \mathbb{R}$ for $\lambda \in \mathbb{S}^{1}$, Equation (i) of Problem (9) is equivalent to Equation (16).

6.2. Solution of the monodromy problem. Without loss of generality, we may (using a Möbius transformation of the sphere) fix the value of $p_{1}, p_{2}$ and $p_{3}$. We still denote $\mathbf{x} \in$ $\left(\mathcal{W}_{\bar{\rho}}^{\geq 0}\right)^{3 n-3}$ the vector of the remaining parameters.

Proposition 2. Assume that the given minimal n-noid is non-degenerate. For $t$ in a neighborhood of 0 , there exists a smooth function $\mathbf{x}(t) \in\left(\mathcal{W}_{\bar{\rho}}^{\geq 0}\right)^{3 n-3}$ such that $\widetilde{M}_{i}(t, \mathbf{x}(t), \cdot) \in \Lambda \mathfrak{s u}(2)$ for $1 \leq i \leq n-1$. Moreover, $\mathbf{x}(0)=\mathbf{x}_{0}$.

Proof: recalling the definition of $P_{i, k}$ in Section 2 and $\mathcal{P}_{i, k}$ in Equation (15), we have

$$
\mathcal{P}_{i, k}(\mathbf{x})(\lambda)=P_{i, k}(\mathbf{x}(\lambda)) \text {. }
$$


Hence $\mathcal{P}_{i, k}$ is a smooth map from a neighborhood of $\mathbf{x}_{0}$ in $\left(\mathcal{W}_{\bar{\rho}}^{\geq 0}\right)^{3 n-3}$ to $\mathcal{W}_{\bar{\rho}}^{\geq 0}$. Moreover, since $\mathbf{x}_{0}$ is constant, we have for $X \in\left(\mathcal{W}_{\bar{\rho}}^{\geq 0}\right)^{3 n-3}$ :

$$
\left(d \mathcal{P}_{i, k}\left(\mathbf{x}_{0}\right) X\right)(\lambda)=d P_{i, k}\left(\mathbf{x}_{0}\right) X(\lambda) .
$$

Let $\mathcal{P}_{i}=\left(\mathcal{P}_{i, 0}, \mathcal{P}_{i, 1}, \mathcal{P}_{i, 2}\right)$ and $\mathcal{P}=\left(\mathcal{P}_{1}, \cdots, \mathcal{P}_{n-1}\right)$. By the non-degeneracy hypothesis and Remark 1. $d P\left(\mathbf{x}_{0}\right)$ is an automorphism of $\mathbb{C}^{3 n-3}$, so $d \mathcal{P}\left(\mathbf{x}_{0}\right)$ is an automorphism of $\left(\mathcal{W}_{\bar{\rho}}^{\geq 0}\right)^{3 n-3}$ and restricts to an automorphism of $\left(\mathcal{W}_{\rho}^{>0}\right)^{3 n-3}$.

We define the following smooth maps with value in $\mathcal{W}_{\rho}$ (the star operator is defined in Section 3.6

$$
\begin{aligned}
& \mathcal{F}_{i}(t, \mathbf{x})=\widetilde{M}_{i, 11}(t, \mathbf{x})+\widetilde{M}_{i, 11}(t, \mathbf{x})^{*} \\
& \mathcal{G}_{i}(t, \mathbf{x})=\widetilde{M}_{i, 12}(t, \mathbf{x})+\widetilde{M}_{i, 21}(t, \mathbf{x})^{*}
\end{aligned}
$$

Problem (16) is equivalent to $\mathcal{F}_{i}=\mathcal{G}_{i}=0$. Actually, by definition, $\mathcal{F}_{i}=\mathcal{F}_{i}^{*}$, so Problem 16 is equivalent to

$$
\mathcal{F}_{i}(t, \mathbf{x})^{+}=0, \quad \operatorname{Re}\left(\mathcal{F}_{i}(t, \mathbf{x})^{0}\right)=0 \quad \text { and } \mathcal{G}_{i}(t, \mathbf{x})=0 \quad \text { for } 1 \leq i \leq n-1 .
$$

At $t=0$, we have by Equation 15$]$ :

$$
\begin{aligned}
& \mathcal{F}_{i}(0, \mathbf{x})=\mathcal{P}_{i, 1}(\mathbf{x})+\mathcal{P}_{i, 1}(\mathbf{x})^{*} \\
& \mathcal{G}_{i}(0, \mathbf{x})=\mathcal{P}_{i, 2}(\mathbf{x})-\mathcal{P}_{i, 0}(\mathbf{x})^{*}
\end{aligned}
$$

Equation (3) tells us precisely that that at the central value, we have $\mathcal{F}_{i}\left(0, \mathbf{x}_{0}\right)=0$ and $\mathcal{G}_{i}\left(0, \mathbf{x}_{0}\right)=$ 0 . We have for $X \in\left(\mathcal{W}_{\bar{\rho}}^{\geq 0}\right)^{3 n-3}$ :

$$
\begin{aligned}
& d \mathcal{F}_{i}\left(0, \mathbf{x}_{0}\right) X=d \mathcal{P}_{i, 1}\left(x_{0}\right) X+\left(d \mathcal{P}_{i, 1}\left(\mathbf{x}_{0}\right) X\right)^{*} \\
& d \mathcal{G}_{i}\left(0, \mathbf{x}_{0}\right) X=d \mathcal{P}_{i, 2}\left(x_{0}\right) X-\left(d \mathcal{P}_{i, 0}\left(\mathbf{x}_{0}\right) X\right)^{*}
\end{aligned}
$$

Projecting on $\mathcal{W}_{\rho}^{>0}$ and $\mathcal{W}_{\rho}^{<0}$ we obtain:

$$
\begin{gathered}
\left(d \mathcal{F}_{i}\left(0, \mathbf{x}_{0}\right) X\right)^{+}=d \mathcal{P}_{i, 1}\left(\mathbf{x}_{0}\right) X^{+} \\
\left(d \mathcal{G}_{i}\left(0, \mathbf{x}_{0}\right) X\right)^{+}=d \mathcal{P}_{i, 2}\left(\mathbf{x}_{0}\right) X^{+} \\
\left(d \mathcal{G}_{i}\left(0, \mathbf{x}_{0}\right) X\right)^{-}=-\left(d \mathcal{P}_{i, 0}\left(\mathbf{x}_{0}\right) X^{+}\right)^{*} \\
\left(d \mathcal{G}_{i}\left(0, \mathbf{x}_{0}\right) X\right)^{-*}=-d \mathcal{P}_{i, 0}\left(\mathbf{x}_{0}\right) X^{+}
\end{gathered}
$$

Hence the operator

$$
\left[d \mathcal{F}_{i}\left(0, \mathbf{x}_{0}\right)^{+}, d \mathcal{G}_{i}\left(0, \mathbf{x}_{0}\right)^{+}, d \mathcal{G}_{i}\left(0, \mathbf{x}_{0}\right)^{-*}\right]_{1 \leq i \leq n-1}
$$

only depends on $X^{+}$and is an automorphism of $\left(\mathcal{W}_{\rho}^{>0}\right)^{3 n-3}$ because $d \mathcal{P}\left(\mathbf{x}_{0}\right)$ is. Projecting on $\mathcal{W}^{0}$ we obtain:

$$
\begin{gathered}
\left(d \mathcal{F}_{i}\left(0, \mathbf{x}_{0}\right) X\right)^{0}=2 \operatorname{Re}\left(d \mathcal{P}_{i, 1}\left(\mathbf{x}_{0}\right) X^{0}\right) \\
\left(d \mathcal{G}_{i}\left(0, \mathbf{x}_{0}\right) X\right)^{0}=d \mathcal{P}_{i, 2}\left(\mathbf{x}_{0}\right) X^{0}-\overline{d \mathcal{P}_{i, 0}\left(\mathbf{x}_{0}\right) X^{0}}
\end{gathered}
$$

Hence the $\mathbb{R}$-linear operator

$$
\left[\operatorname{Re}\left(d \mathcal{F}_{i}\left(0, \mathbf{x}_{0}\right)^{0}\right), d \mathcal{G}_{i}\left(0, \mathbf{x}_{0}\right)^{0}\right]_{1 \leq i \leq n-1}
$$

only depends on $X^{0}$ and is surjective from $\mathbb{C}^{3 n-3}$ to $(\mathbb{R} \times \mathbb{C})^{3 n-3}$. This implies that the differential of the map $\left(\mathcal{F}_{i}^{+}, \mathcal{G}_{i}^{+}, \mathcal{G}_{i}^{-*}, \operatorname{Re}\left(\mathcal{F}_{i}^{0}\right), \mathcal{G}_{i}^{0}\right)_{1 \leq i \leq n-1}$ is surjective from $\left(\mathcal{W}_{\bar{\rho}}^{\geq 0}\right)^{3 n-3}$ to $\left(\left(\mathcal{W}_{\rho}^{>0}\right)^{3} \times \mathbb{R} \times\right.$ $\mathbb{C})^{n-1}$. Proposition 2 follows from the Implicit Function Theorem. 
Remark 3. The kernel of the differential has real dimension $3 n-3$ so we have $3 n-3$ free real parameters. These parameters correspond to deformations of the flux vectors of the minimal n-noid.

\section{Geometry of the immersion}

From now on, we assume that $\mathbf{x}(t)$ is given by Proposition 2. We write $a_{i, t}, b_{i, t}$ and $p_{i, t}$ for the value of the corresponding parameters. (These parameters are in the space $\mathcal{W}_{\bar{\rho}}^{\geq 0}$ so are functions of $\lambda$.) For ease of notation, we write $g_{t}, \omega_{t}, \xi_{t}$ and $\Phi_{t}$ for $g_{\mathbf{x}(t)}, \omega_{\mathbf{x}(t)}, \xi_{t, \mathbf{x}(t)}$ and $\Phi_{t, \mathbf{x}(t)}$, respectively. Let $F_{t}=\operatorname{Uni}\left(\Phi_{t}\right)$. Since the Monodromy Problem is solved, the Sym-Bobenko formula (6) defines a CMC-1 immersion $f_{t}: \Omega \rightarrow \mathbb{R}^{3}$, where $\Omega$ is the (fixed) domain defined by Equation (13).

Proposition 3. The immersion $f_{t}$ extends analytically to

$$
\Sigma_{t}:=\mathbb{C} \cup\{\infty\} \backslash\left\{p_{1, t}(0), \cdots, p_{n, t}(0)\right\}
$$

where $p_{i, t}(0)$ is the value of $p_{i, t}$ at $\lambda=0$.

We omit the proof which is exactly the same as the proof of Point 1 of Proposition 4 in [14. It relies on Theorem 3 in 14 which allows for $\lambda$-dependent changes of variables in the DPW method.

\subsection{Convergence to the minimal $n$-noid.}

Proposition 4. $\lim _{t \rightarrow 0} \frac{1}{t} f_{t}=\psi$ where $\psi$ is (up to translation) the conformal parametrization of the minimal n-noid given by Equation (1). The limit is the uniform $C^{1}$ convergence on compact subsets of $\Sigma_{0}=\mathbb{C} \cup\{\infty\} \backslash\left\{p_{1,0}, \cdots, p_{n, 0}\right\}$.

Proof: at $t=0$, we have $g_{0}=g$ and $\omega_{0}=\omega$. By Equation (12) and definition of the potential, we have

$$
\Phi_{0}(z, \lambda)=\left(\begin{array}{cc}
g(z) & 1 \\
-1 & 0
\end{array}\right) \quad \text { and }\left.\quad \frac{\partial \xi_{t ; 12}^{(-1)}}{\partial t}\right|_{t=0}=\frac{\omega}{4}
$$

By Theorem 4, $\frac{1}{t} f_{t}$ converges to a minimal surface with Weierstrass data $(g, \omega)$ on compact subsets of $\Sigma_{0}$ minus the poles of $g$. In a neighborhood of the poles of $g$, we use the gauge introduced in Section 5.1. With the notations of this section and writing $\widehat{\Phi}_{t}=\Phi_{t} G_{\mathbf{x}(t)}$, we have

$$
\widehat{\Phi}_{0}(z, \lambda)=\left(\begin{array}{cc}
1 & 0 \\
-g(z)^{-1} & 1
\end{array}\right) \quad \text { and }\left.\quad \frac{\partial \widehat{\xi}_{t ; 12}^{(-1)}}{\partial t}\right|_{t=0}=\frac{g^{2} \omega}{4} .
$$

By Theorem 4 again, $\frac{1}{t} f_{t}$ converges to a minimal surface with Weierstrass data $(g, \omega)$ in a neighborhood of the poles of $g$. The two limit minimal surfaces are of course the same, since they coincide in a neighborhood of $z_{0}$.

7.2. Delaunay ends. We denote $N_{0}$ the Gauss map of the minimal $n$-noid $M_{0}$. For $1 \leq i \leq n$, we denote $\mathcal{C}_{i}$ the catenoid to which $M_{0}$ is asymptotic at $p_{i, 0}$ and $\tau_{i}>0$ the necksize of $\mathcal{C}_{i}$.

Definition 4. We say that $N_{0}$ points to the inside in a neighborhood of $p_{i, 0}$ if it points to the component of $\mathbb{R}^{3} \backslash \mathcal{C}_{i}$ containing the axis of $\mathcal{C}_{i}$.

Proposition 5. For $1 \leq i \leq n$ and $t \neq 0$ : 
(1) The immersion $f_{t}$ has a Delaunay end at $p_{i, t}$. If we denote $w_{i, t}$ its weight then

$$
\lim _{t \rightarrow 0} t^{-1} w_{i, t}= \pm 2 \pi \tau_{i}
$$

where the sign is + if $N_{0}$ points to the inside in a neighborhood of $p_{i, 0}$ and - otherwise.

(2) Its axis converges as $t \rightarrow 0$ to the half-line through the origin directed by the vector $N_{0}\left(p_{i, 0}\right)$.

(3) If $N_{0}$ points to the inside in a neighborhood of $p_{i, 0}$, there exists a uniform $\varepsilon>0$ such that for $t>0$ small enough, $f_{t}\left(D^{*}\left(p_{i, 0}, \varepsilon\right)\right)$ is embedded.

Proof: in a neighborhood of the puncture $p_{i, t}$, we may use $w=g_{t}(z)-g_{t}\left(p_{i, t}\right)$ as a local coordinate. (This change of coordinate depends on $\lambda$. This is not a problem by Theorem 3 in [14].) Consider the gauge

$$
G(w)=\left(\begin{array}{cc}
\frac{k}{\sqrt{w}} & \frac{-1}{2 k \sqrt{w}} \\
0 & \frac{\sqrt{w}}{k}
\end{array}\right) .
$$

Here we can take $k=1$, but later on we will take another value of $k$ so we do the computation for general values of $k \neq 0$. The gauged potential is

$$
\widehat{\xi}_{t}:=\xi_{t} \cdot G=\left(\begin{array}{cc}
0 & \frac{d w}{4 k^{2} w}+\frac{w t(\lambda-1)^{2}}{4 k^{2} \lambda} \omega_{t} \\
\frac{k^{2} d w}{w} & 0
\end{array}\right)
$$

Since $\omega_{t}$ has a double pole at $p_{i, t}, \widehat{\xi}_{t}$ has a simple pole at $w=0$ with residue

$$
A_{i, t}(\lambda)=\left(\begin{array}{cc}
0 & \frac{1}{4 k^{2}}+\frac{t(\lambda-1)^{2}}{4 k^{2} \lambda} \alpha_{i, t}(\lambda) \\
k^{2} & 0
\end{array}\right)
$$

where

$$
\alpha_{i, t}=\operatorname{Res}_{p_{i, t}}\left(w \omega_{t}\right)=\operatorname{Res}_{p_{i, t}}\left(g_{t}(z)-g_{t}\left(p_{i, t}\right)\right) \omega_{t} .
$$

Claim 1. For $t$ small enough, $\alpha_{i, t}$ is a real constant (i.e. independent of $\lambda$, possibly depending on $t$ ).

Proof: the proof is similar to the proof of Point 2 of Proposition 4 in [14. We use the standard theory of Fuchsian systems. Fix $t \neq 0$ and $\lambda \in \mathbb{S}^{1} \backslash\{1\}$. Assume that $\alpha_{i, t}(\lambda) \neq 0$. Let $\widehat{\Phi}_{t}=\Phi_{t} G$. The eigenvalues of $A_{i, t}$ are $\pm \Lambda_{i, t}$ with

$$
\Lambda_{i, t}(\lambda)^{2}=\frac{1}{4}+\frac{t(\lambda-1)^{2}}{4 \lambda} \alpha_{i, t}(\lambda)
$$

Provided $t \neq 0$ is small enough, $\Lambda_{i, t} \notin \mathbb{Z} / 2$ so the system is non resonant and $\widehat{\Phi}_{t}$ has the following standard $z^{A} P$ form in the universal cover of $D(0, \varepsilon)^{*}$ :

$$
\widehat{\Phi}_{t}(w, \lambda)=V(\lambda) \exp \left(A_{i, t}(\lambda) \log w\right) P(w, \lambda)
$$

where $P(w, \lambda)$ descends to a well defined holomorphic function of $w \in D(0, \varepsilon)$ with $P(0, \lambda)=I_{2}$. Consequently, its monodromy is

$$
\mathcal{M}_{\gamma_{i}}\left(\widehat{\Phi}_{t}\right)=V(\lambda) \exp \left(2 \pi \mathrm{i} A_{i, t}\right) V(\lambda)^{-1}
$$

with eigenvalues $\exp \left( \pm 2 \pi \mathrm{i} \Lambda_{i, t}(\lambda)\right)$. Since the Monodromy Problem is solved, the eigenvalues are unitary complex numbers, so $\Lambda_{i, t}(\lambda) \in \mathbb{R}$ which implies that $\alpha_{i, t}(\lambda) \in \mathbb{R}$. This of course remains 
true if $\alpha_{i, t}(\lambda)=0$. Hence $\alpha_{i, t}$ is real on $\mathbb{S}^{1} \backslash\{1\}$. Since all the parameters involved in the definition of $\omega_{t}$ are in $\mathcal{W}_{\bar{\rho}}^{\geq 0}, \alpha_{i, t}$ is holomorphic in the unit disk. Hence it is constant.

Returning to the proof of Proposition 5 , let $(r, s) \in \mathbb{R}^{2}$ be the solution of

$$
\left\{\begin{array}{l}
r s=\frac{1}{4} t \alpha_{i, t} \\
r+s=\frac{1}{2} \\
r<s
\end{array}\right.
$$

Since $r<s, \sqrt{r \lambda+s}$ is well defined and does not vanish for $\lambda \in \mathbb{D}$. We take $k=\sqrt{r \lambda+s}$ in the definition of the gauge $G$. Using Equation (20), we have:

$$
\left(r \lambda^{-1}+s\right)(r \lambda+s)=\frac{1}{4}+r s(\lambda-1)^{2} \lambda^{-1}=\frac{1}{4}+\frac{1}{4} t(\lambda-1)^{2} \lambda^{-1} \alpha_{i, t} .
$$

So the residue of $\widehat{\xi}_{t}$ becomes

$$
A_{i, t}=\left(\begin{array}{cc}
0 & \frac{1}{r \lambda+s}\left(\frac{1}{4}+\frac{t(\lambda-1)^{2}}{4 \lambda} \alpha_{i, t}\right) \\
r \lambda+s & 0
\end{array}\right)=\left(\begin{array}{cc}
0 & r \lambda^{-1}+s \\
r \lambda+s & 0
\end{array}\right)
$$

which is the residue of the standard Delaunay potential. By [9], the immersion $f_{t}$ has a Delaunay end at $p_{i, t}$ of weight $w_{i, t}=8 \pi r s=2 \pi t \alpha_{i, t}$. It remains to relate $\alpha_{i, 0}$ to the logarithmic growth $\tau_{i}$. For ease of notation, let us write $p_{i}=p_{i, 0}$. Assume that $N_{0}$ points to the inside in a neighborhood of $p_{i}$. The flux of $M_{0}$ along $\gamma_{i}$ is equal to

$$
\phi_{i}=2 \pi \tau_{i} N_{0}\left(p_{i}\right)=2 \pi \frac{\tau_{i}}{\left|g\left(p_{i}\right)\right|^{2}+1}\left(2 \operatorname{Re}\left(g\left(p_{i}\right)\right), 2 \operatorname{Im}\left(g\left(p_{i}\right)\right),\left|g\left(p_{i}\right)\right|^{2}-1\right)
$$

On the other hand, we have seen in Section 2 that the flux is equal to

$$
\phi_{i}=-2 \pi \operatorname{Res}_{p_{i}}\left(\frac{1}{2}\left(1-g^{2}\right) \omega, \frac{\mathrm{i}}{2}\left(1+g^{2}\right) \omega, g \omega\right)
$$

Comparing these two expressions for $\phi_{i}$, we obtain

$$
\operatorname{Res}_{p_{i}}(g \omega)=-\tau_{i} \frac{\left|g\left(p_{i}\right)\right|^{2}-1}{\left|g\left(p_{i}\right)\right|^{2}+1} \quad \text { and } \quad \operatorname{Res}_{p_{i}} \omega=-2 \tau_{i} \frac{\overline{g\left(p_{i}\right)}}{\left|g\left(p_{i}\right)\right|^{2}+1}
$$

Using Equation 190 , this gives

$$
\alpha_{i, 0}=\operatorname{Res}_{p_{i}}(g \omega)-g\left(p_{i}\right) \operatorname{Res}_{p_{i}} \omega=\tau_{i}
$$

If $N_{0}$ points to the outside in a neighborhood of $p_{i}$, then $\phi_{i}=-2 \pi \tau_{i} N_{0}\left(p_{i}\right)$, so the same computation gives $\alpha_{i, 0}=-\tau_{i}$. This proves Point 1 of Proposition 5

To prove Point 2, we use Theorem 6 in Appendix B. We need to compute $\widehat{\Phi}_{0}$ at $w=1$. At $t=0$, we have $k=\frac{1}{\sqrt{2}}$ so

$$
G(1)=\frac{1}{\sqrt{2}}\left(\begin{array}{cc}
1 & -1 \\
0 & 2
\end{array}\right)
$$

At $t=0$, we have $w=g(z)-g\left(p_{i}\right)$, so $w=1 \Leftrightarrow g(z)=g\left(p_{i}\right)+1$. Using Equation 12,

$$
\widehat{\Phi}_{0}(1)=\frac{1}{\sqrt{2}}\left(\begin{array}{cc}
g\left(p_{i}\right)+1 & 1 \\
-1 & 0
\end{array}\right)\left(\begin{array}{cc}
1 & -1 \\
0 & 2
\end{array}\right)=\frac{1}{\sqrt{2}}\left(\begin{array}{cc}
g\left(p_{i}\right)+1 & -g\left(p_{i}\right)+1 \\
-1 & 1
\end{array}\right) .
$$

Fix $0<\alpha<1$. By Theorem 6 (using $t \alpha_{i, t}$ as the time parameter), there exists $\varepsilon>0, T>0$ and $c$ such that for $0<|t|<T$ :

$$
\left\|f_{t}(z)-f_{i, t}^{\mathcal{D}}(z)\right\| \leq c|t|\left|z-p_{i, t}\right|^{\alpha} \quad \text { in } D^{*}\left(p_{i, t}, \varepsilon\right)
$$


where $f_{i, t}^{\mathcal{D}}: \mathbb{C} \backslash\left\{p_{i, t}\right\} \rightarrow \mathbb{R}^{3}$ is a Delaunay immersion. We compute the limit axis of $f_{i, t}^{\mathcal{D}}$ using Point 3 of Theorem 6:

$$
\begin{gathered}
\widehat{\Phi}_{0}(1) H=\left(\begin{array}{cc}
g\left(p_{i}\right) & 1 \\
-1 & 0
\end{array}\right)=\Phi_{0}\left(p_{i}\right) . \\
Q=F_{0}\left(p_{i}\right) \\
Q e_{3} Q^{-1}=\operatorname{Nor}\left(F_{0}\left(p_{i}\right)\right)=N_{0}\left(p_{i}\right) .
\end{gathered}
$$

This proves Point 2 of Proposition 5. If $N_{0}$ points to the inside in a neighborhood of $p_{i, 0}$, then for $t>0, t \alpha_{i, t}>0$ so Point 3 follows from Point 2 of Theorem 6 .

7.3. Alexandrov-embeddedness. We recall from [2, 6] the definition of Alexandrov-embeddedness in the non-compact case:

Definition 5. A surface $M$ of finite topology is Alexandrov-embedded if $M$ is properly immersed, if each end of $M$ is embedded, and if there exists a compact 3-manifold $\bar{W}$ with boundary $\partial \bar{W}=\bar{S}$, $n$ points $q_{1}, \cdots, q_{n} \in \bar{S}$ and a proper immersion $F: W=\bar{W} \backslash\left\{q_{1}, \cdots, q_{n}\right\} \rightarrow \mathbb{R}^{3}$ whose restriction to $S=\bar{S} \backslash\left\{q_{1}, \cdots, q_{n}\right\}$ parametrizes $M$.

Let $M$ be an Alexandrov-embedded minimal surface with $n$ catenoidal ends. With the notations of Definition 5, we equip $W$ with the flat metric induced by $F$, so $F$ is a local isometry. We denote $N$ the inside normal to $S$.

Lemma 1. There exists a flat 3-manifold $W^{\prime}$ containing $W$, a local isometry $F^{\prime}: W^{\prime} \rightarrow \mathbb{R}^{3}$ extending $F$ and $r>0$ such that the tubular neighborhood $T u b_{r} S$ is embedded in $W^{\prime}$. In other words, the map $(x, s) \mapsto \exp _{x}(s N(x))$ from $S \times(-r, r)$ to $W^{\prime}$ is well defined and is a diffeomorphism onto its image.

Proof: since $M$ has catenoidal ends, there exists $r>0$ such that the inside tubular neighborhood map

$$
g: S \times(0, r) \rightarrow W, \quad g(x, s)=\exp _{x}(s N(x))
$$

is a diffeomorphism onto its image. Since $F$ is a local isometry, we have

$$
F(g(x, s))=F(x)+s d F(x) N(x) \quad \text { for }(x, s) \in S \times(0, r) .
$$

We define $W^{\prime}$ as the disjoint union $(S \times(-r, r)) \sqcup W$ where we identify $(x, s) \in S \times(0, r)$ with its image $g(x, s) \in W$. We define $F^{\prime}: W^{\prime} \rightarrow \mathbb{R}^{3}$ by $F^{\prime}=F$ in $W$ and

$$
F^{\prime}(x, s)=F(x)+s d F(x) N(x) \quad \text { for }(x, s) \in S \times(-r, r) .
$$

The map $F^{\prime}$ is well defined by Equation (21). We equip $S \times(-r, r)$ with the flat metric induced by the local diffeomorphism $F^{\prime}$, which extends the metric already defined on $S \times(0, r)$ by identification with $W$. Since

$$
d F^{\prime}(x, 0)(X, T)=d F(x) X+T d F(x) N(x)
$$

the metric restricted to $S \times\{0\}$ is the product metric, so the normal to $S \times\{0\}$ in $S \times(-r, r)$ is $N(x, 0)=(0,1)$. Since $F^{\prime}$ is a local isometry, we have for $(x, s) \in S \times(-r, r)$

$$
F^{\prime}\left(\exp _{(x, 0)} s N(x, 0)\right)=F^{\prime}(x, 0)+s d F^{\prime}(x, 0)(0,1)=F(x)+s d F(x) N(x)=F^{\prime}(x, s)
$$

Hence $\exp _{(x, 0)} s N(x, 0)=(x, s)$ so $\operatorname{Tub}_{r}(S \times\{0\})$ is embedded in $S \times(-r, r)$. 


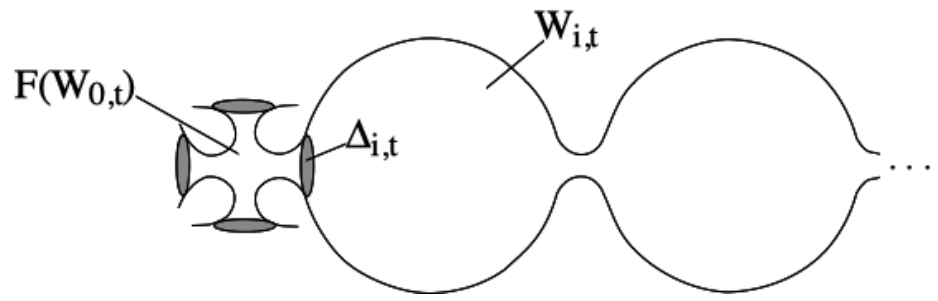

Figure 2. Decomposition of a 4-noid into pieces. Only one Delaunay end is represented, and $F\left(W_{0, t}\right)$ is represented as an embedded domain for clarity, but in general it will be immersed.

We now return to the proof of Theorem 1 . We orient the minimal $n$-noid $M_{0}$ so that its Gauss map points to the inside in a neighborhood of $p_{1}$. For $0<|t|<\epsilon$, we denote $M_{t}$ the image of the immersion $f_{t}$ that we have constructed.

Proposition 6. If $M_{0}$ is Alexandrov embedded, then for $t>0$ small enough, $M_{t}$ is Alexandrov embedded.

Proof: our strategy is to cut $M_{t}$ by suitable planes into pieces which are either close to $M_{0}$ or Delaunay surfaces (see Figure 2). Then we prove that each piece, together with flat disks in the cutting planes, is the boundary of a domain, using the Jordan Brouwer Theorem.

Since $M_{0}$ is Alexandrov embedded, $N_{0}$ points to the inside in a neighborhood of each end, so $M_{t}$ has embedded ends by Proposition 5. Let $\varepsilon>0$ be the number given by our application of Theorem 6 in Section 7.2 and $f_{i, t}^{\mathcal{D}}: \mathbb{C} \backslash\left\{p_{i, t}\right\} \rightarrow \mathbb{R}^{3}$ be the Delaunay immersion which approximates $f_{t}$ in $D^{*}\left(p_{i, t}, \varepsilon\right)$. Recall that $f_{t}\left(D^{*}\left(p_{i, t}, \varepsilon\right)\right)$ is embedded. Let $\widetilde{f}_{t}=\frac{1}{t} f_{t}$. By Proposition 4, $\widetilde{f}_{t}$ converges to $\psi$ on compact subsets of $\Sigma_{0}$, where $\psi: \Sigma_{0} \rightarrow \mathbb{R}^{3}$ is a parametrization of $M_{0}$. Since $M_{0}$ has catenoidal ends, we may assume (taking $\varepsilon$ smaller if necessary) that $\psi\left(D^{*}\left(p_{i, 0}, \varepsilon\right)\right)$ is embedded and $N_{0} \neq N_{0}\left(p_{i, 0}\right)$ in $D^{*}\left(p_{i, 0}, \varepsilon\right)$.

Let $h_{i}: \mathbb{R}^{3} \rightarrow \mathbb{R}$ be the height function in the direction $N_{0}\left(p_{i, 0}\right)$, defined by

$$
h_{i}(x)=\left\langle x, N_{0}\left(p_{i, 0}\right)\right\rangle .
$$

We shall cut $M_{t}$ by the plane $h_{i}=\delta$ where $\delta>0$ is a fixed, large enough number such that for $1 \leq i \leq n$,

$$
\delta>\max _{C\left(p_{i, 0}, \varepsilon\right)} h_{i} \circ \psi .
$$

Since $\lim _{z \rightarrow p_{i, 0}} h_{i} \circ \psi(z)=+\infty$, we may fix a positive, small enough $\varepsilon^{\prime}<\varepsilon$ such that

$$
\min _{C\left(p_{i, 0}, \varepsilon^{\prime}\right)} h_{i} \circ \psi>\delta .
$$

Let $\mathcal{A}_{i, t}$ be the annulus defined by $\varepsilon^{\prime} \leq\left|z-p_{i, t}\right| \leq \varepsilon$. Since $N_{0} \neq N_{0}\left(p_{i, 0}\right)$ in $\mathcal{A}_{i, 0}$,

$$
\min _{\mathcal{A}_{i, 0}}\left\|N_{0}(z)-N_{0}\left(p_{i, 0}\right)\right\|>0 \text {. }
$$

For $t>0$ small enough:

$$
\max _{C\left(p_{i, t}, \varepsilon\right)} h_{i} \circ \widetilde{f}_{t}<\delta
$$




$$
\begin{gathered}
\min _{C\left(p_{i, t}, \varepsilon^{\prime}\right)} h_{i} \circ \widetilde{f}_{t}>\delta \\
\min _{\mathcal{A}_{i, t}}\left\|N_{t}(z)-N_{0}\left(p_{i, 0}\right)\right\|>0 .
\end{gathered}
$$

Hence the function $h_{i} \circ \widetilde{f}_{t}$ has no critical point in the annulus $\mathcal{A}_{i, t}$. So $h_{i} \circ \widetilde{f}_{t}=\delta$ defines a regular closed curve $\gamma_{i, t}$ in $\mathcal{A}_{i, t}$. At $t=0, h_{i} \circ \psi=\delta$ is a single curve around $p_{i, 0}$, so $\gamma_{i, t}$ has only one component and is not contractible in $\mathcal{A}_{i, t}$. Let $D_{i, t} \subset \mathbb{C}$ be the topological disk bounded by $\gamma_{i, t}$ and $D_{i, t}^{*}=D_{i, t} \backslash\left\{p_{i, t}\right\}$. Let $\Delta_{i, t}$ be the closed topological disk bounded by $\widetilde{f}_{t}\left(\gamma_{i, t}\right)$ in the plane defined by $h_{i}(x)=\delta$.

Claim 2. For $t>0$ small enough, $\widetilde{f}_{t}\left(D_{i, t}^{*}\right) \cap \Delta_{i, t}=\emptyset$.

Proof: of course, $h_{i} \circ \widetilde{f}_{t}>\delta$ in $D_{i, t}^{*} \cap \mathcal{A}_{i, t}$. What we need to prove is that $\widetilde{f}_{t}\left(D^{*}\left(p_{i, t}, \varepsilon^{\prime}\right)\right)$ does not intersect $\Delta_{i, t}$. We do this by comparison with the Delaunay surface. Let $\Pi_{i}=N_{0}\left(p_{i, 0}\right)^{\perp}$ and $\pi_{i}=\mathbb{R}^{3} \rightarrow \Pi_{i}$ be the orthogonal projection. Since $\psi$ has a catenoidal end at $p_{i, 0}, \psi\left(\mathcal{A}_{i, t}\right)$ is a graph over an annulus in the plane $\Pi_{i}$, with inside boundary circle $\pi_{i} \circ \psi\left(C\left(p_{i, t}, \varepsilon\right)\right)$ and outside boundary circle $\pi_{i} \circ \psi\left(C\left(p_{i, t}, \varepsilon^{\prime}\right)\right)$. Moreover, $N_{0}$ is close to $N_{0}\left(p_{i, t}\right)$. Since $\widetilde{f}_{t}$ is $C^{1}$ close to $\psi$ in $\mathcal{A}_{i, t}$, for $t>0$ small enough, $\widetilde{f}_{t}\left(\mathcal{A}_{i, t}\right)$ is a graph over an annulus in the plane $\Pi_{i}$, with inside boundary circle $\pi_{i} \circ \widetilde{f}_{t}\left(C\left(p_{i, t}, \varepsilon\right)\right)$ and outside boundary circle $\pi_{i} \circ \widetilde{f}_{t}\left(C\left(p_{i, t}, \varepsilon^{\prime}\right)\right)$.

Now we go back to the original scale. Since $f_{t}$ is $C^{1}$ close to $f_{i, t}^{\mathcal{D}}$ in $D^{*}\left(p_{i, t}, \varepsilon\right)$, we conclude that $f_{i, t}^{\mathcal{D}}\left(\mathcal{A}_{i, t}\right)$ is a graph over an annulus in the plane $\Pi_{i}$, with inside boundary circle $\pi_{i} \circ f_{i, t}^{\mathcal{D}}\left(C\left(p_{i, t}, \varepsilon\right)\right)$ and outside boundary circle $\pi_{i} \circ f_{i, t}^{\mathcal{D}}\left(C\left(p_{i, t}, \varepsilon^{\prime}\right)\right)$. Then from the geometry of Delaunay surfaces, there exists a curve $\gamma_{i, t, 0}$ in $D^{*}\left(p_{i, t}, \varepsilon^{\prime}\right)$ such that $f_{i, t}^{\mathcal{D}}\left(\gamma_{i, t, 0}\right)$ is a closed curve in the plane $h_{i}=\frac{1}{2}$. Let $D_{i, t, 0}$ be the disk bounded by $\gamma_{i, t, 0}$ and $\mathcal{A}_{i, t, 0}$ be the closed annulus bounded by $\gamma_{i, t}$ and $\gamma_{i, t, 0}$. Then $h_{i} \circ f_{i, t}^{\mathcal{D}}>\frac{1}{2}$ in $D_{i, t, 0}$ and $f_{i, t}^{\mathcal{D}}\left(\mathcal{A}_{i, t, 0}\right)$ is a graph over an annulus in the plane $\Pi_{i}$. Since $f_{t}$ is $C^{1}$ close to $f_{i, t}^{\mathcal{D}}$ in $D^{*}\left(p_{i, t}, \varepsilon\right)$, we conclude that $h_{i} \circ f_{t}>\frac{1}{4}$ in $D_{i, t, 0}^{*}$ and $f_{t}\left(\mathcal{A}_{i, t, 0}\right)$ is a graph over an annulus in the plane $\Pi_{i}$.

Back to the scale $\frac{1}{t}, \widetilde{f}_{t}\left(D_{i, t} \cap \mathcal{A}_{i, t, 0}\right)$ is a graph over an annulus in the plane $\Pi_{i}$ whose inside boundary circle is $\pi_{i} \circ \widetilde{f}_{t}\left(\gamma_{i, t}\right)=\pi_{i}\left(\partial \Delta_{i, t}\right)$, so $\widetilde{f}_{t}\left(D_{i, t} \cap \mathcal{A}_{i, t, 0}\right) \cap \Delta_{i, t}=\emptyset$. Moreover, $h_{i} \circ \widetilde{f}_{t}>\frac{1}{4 t} \gg \delta$ in $D_{i, t, 0}^{*}$ so $\widetilde{f}_{t}\left(D_{i, t, 0}^{*}\right) \cap \Delta_{i, t}=\emptyset$.

Claim 3. For $t>0$ small enough, $\widetilde{f}_{t}\left(D_{i, t}^{*}\right) \cup \Delta_{i, t}$ is the boundary of a cylindrically bounded domain $W_{i, t} \subset \mathbb{R}^{3}$.

Proof: since $f_{t}$ is close to $f_{i, t}^{\mathcal{D}}$ in $D_{i, t}^{*}$, we can find an increasing diverging sequence $\left(R_{t, k}\right)_{k \in \mathbb{N}}$ such that $f_{t}\left(D_{i, t}^{*}\right)$ intersects the plane $h_{i}=R_{t, k}$ transversally along a closed curve $f_{t}\left(\gamma_{i, t, k}\right)$. (Explicitely, we can take $R_{t, k}=\frac{1}{2}+k h_{i}\left(T_{t}\right)$ where $T_{t} \in \mathbb{R}^{3}$ is the period of the Delaunay surface $f_{i, t}^{\mathcal{D}}$.) Let $\mathcal{A}_{i, t, k}$ be the annulus bounded by $\gamma_{i, t}$ and $\gamma_{i, t, k}$. Let $\Delta_{i, t, k}$ be the closed disk bounded by $\widetilde{f}_{t}\left(\gamma_{i, t, k}\right)$ in the plane $h_{i}=t^{-1} R_{t, k}$. Then $\widetilde{f}_{t}\left(\mathcal{A}_{i, t, k}\right) \cup \Delta_{i, t} \cup \Delta_{i, t, k}$ is topologically a sphere: the image of $\mathbb{S}^{2}$ by an injective continuous map. By the Jordan Brouwer Theorem, it is the boundary of a bounded domain $W_{i, t, k}$. Clearly, $W_{i, t, k} \subset W_{i, t, k+1}$. We take $W_{i, t}=\bigcup_{k \in \mathbb{N}} W_{i, t, k}$. 
Let $\Omega_{t}=\mathbb{C} \cup\{\infty\} \backslash\left(D_{1, t} \cup \cdots \cup D_{n, t}\right)$. Let $W^{\prime}$ be the flat 3-manifold given by Lemma 1 and denote $F: W^{\prime} \rightarrow \mathbb{R}^{3}$ its developing map (instead of $F^{\prime}$ ). (Here $W^{\prime}$ is an open manifold, meaning not a manifold-with-boundary.)

Claim 4. For $t>0$ small enough, there exists a compact domain $W_{0, t}$ in $W^{\prime}$ such that

$$
F\left(\partial W_{0, t}\right)=\widetilde{f}_{t}\left(\Omega_{t}\right) \cup \Delta_{1, t} \cup \cdots \cup \Delta_{n, t} .
$$

Proof: by definition, $\psi$ lifts to a diffeomorphism $\widehat{\psi}: \Sigma_{0} \rightarrow S \subset W^{\prime}$ such that $F \circ \widehat{\psi}=\psi$. Since $M_{0}$ has catenoidal ends, there exists domains $V_{1}, \cdots, V_{n}$ in $W^{\prime}$ such that for $1 \leq i \leq n$ :

- $F: V_{i} \rightarrow F\left(V_{i}\right) \subset \mathbb{R}^{3}$ is a diffeomorphism,

- $V_{i}$ is foliated by flat disks on which $h_{i} \circ F$ is constant (in particular, $h_{i} \circ F$ is constant on $\left.\partial V_{i}\right)$,

- $\widehat{\psi}\left(D^{*}\left(p_{i, 0}, \varepsilon\right)\right) \subset V_{i}$ (which might require taking a smaller $\varepsilon>0$ ),

- $h_{i}<\delta$ on $V_{i} \cap \widehat{\psi}\left(\Sigma_{0} \backslash \bigcup_{i=1}^{n} D\left(p_{i, 0}, \varepsilon\right)\right.$ ) (which might require taking a larger $\delta$ ).

Let $r>0$ be the radius of the embedded tubular neighborhood of $S$ in $W^{\prime}$ constructed in Lemma 1. For $t>0$ small enough, $\left\|\widetilde{f}_{t}-\psi\right\|<r$ in $\bar{\Omega}_{t}$, so $\widetilde{f}_{t}$ lifts to $\widehat{f}_{t}: \bar{\Omega}_{t} \rightarrow W^{\prime}$ such that $F \circ \widehat{f}_{t}=\widetilde{f}_{t}$. (Explicitely, $\widehat{f}_{t}(z)=\exp _{\psi(z)}\left(\widetilde{f}_{t}(z)-\psi(z)\right)$.) From the properties of $V_{i}$ and the convergence of $\widehat{f}_{t}$ to $\widehat{\psi}$ on compact subsets of $\Sigma_{0}$, we have for $t>0$ small enough

$$
\begin{gathered}
\widehat{f}_{t}\left(\bar{\Omega}_{t} \cap D\left(p_{i, 0}, \varepsilon\right)\right) \subset V_{i} \\
h_{i}<\delta \quad \text { on } V_{i} \cap \widehat{f}_{t}\left(\Sigma_{0} \backslash \bigcup_{i=1}^{n} D\left(p_{i, 0}, \varepsilon\right)\right) .
\end{gathered}
$$

By Equation (25), $\widehat{f}_{t}\left(\gamma_{i, t}\right) \subset V_{i}$ so $\Delta_{i, t}$ lifts to a closed disk $\widehat{\Delta}_{i, t} \subset V_{i}$ such that $\partial \widehat{\Delta}_{i, t}=\widehat{f}_{t}\left(\gamma_{i, t}\right)$ and $F\left(\widehat{\Delta}_{i, t}\right)=\Delta_{i, t}$. Since $F$ is a diffeomorphism on $V_{i}, \widehat{f}_{t}\left(\Omega_{t} \cap D\left(p_{i, t}, \varepsilon\right)\right)$ is disjoint from $\widehat{\Delta}_{i, t}$. By (26), $\widehat{f}_{t}\left(\Omega_{t} \backslash \bigcup_{i=1}^{n} D\left(p_{i, t}, \varepsilon\right)\right)$ is disjoint from $\widehat{\Delta}_{i, t}$. Hence $\widehat{f}_{t}\left(\Omega_{t}\right) \cap \widehat{\Delta}_{i, t}=\emptyset$. Then $\widehat{f}_{t}\left(\Omega_{t}\right) \cup \widehat{\Delta}_{1, t} \cup \cdots \cup \widehat{\Delta}_{n, t}$ is a topological sphere in $W^{\prime}$. Since $M_{0}$ has genus zero, $W^{\prime}$ is homeomorphic to $\mathbb{R}^{3}$. By the Jordan Brouwer Theorem, $\widehat{f}_{t}\left(\Omega_{t}\right) \cup \widehat{\Delta}_{1, t} \cup \cdots \cup \widehat{\Delta}_{n, t}$ is the boundary of a compact domain $W_{0, t} \subset W^{\prime}$.

Returning to the proof of Proposition 6, let $W_{t}$ be the abstract 3-manifold with boundary obtained as the disjoint union $\bar{W}_{0, t} \sqcup \bar{W}_{1, t} \sqcup \cdots \sqcup \bar{W}_{n, t}$, identifying $\bar{W}_{0, t}$ and $\bar{W}_{i, t}$ along their boundaries $\widehat{\Delta}_{i, t}$ and $\Delta_{i, t}$ via the map $F$ for $1 \leq i \leq n$. Let $F_{t}: W_{t} \rightarrow \mathbb{R}^{3}$ be the map defined by $F_{t}=F$ in $\bar{W}_{0, t}$ and $F_{t}=$ id in $\bar{W}_{i, t}$ for $1 \leq i \leq n$. Then $F_{t}$ is a proper local diffeomorphism whose boundary restriction parametrizes $M_{t}$. Moreover, since each $\bar{W}_{i, t}$ is homeomorphic to a closed ball minus a boundary point, we may compactify $W_{t}$ by adding $n$ points. This proves that $M_{t}$ is Alexandrov-embedded.

\section{Appendix A. Appendix: complements on the Banach algebra $\mathcal{W}_{\rho}$}

In this section, we prove several basic facts about the Banach algebra $\mathcal{W}_{\rho}$ introduced in Section 3.6 that are used in this paper and related papers [14, 15].

Proposition 7. If $\rho \geq 1, \mathcal{W}_{\rho}$ is a Banach algebra. 
Proof: let $f, g \in \mathcal{W}_{\rho}$ and $h=f g$. Then $\left(\left|f_{i}\right| \rho^{|i|}\right)_{i \in \mathbb{Z}}$ and $\left(\left|g_{i}\right| \rho^{|i|}\right)_{i \in \mathbb{Z}}$ are summable families, so $\left(\left|f_{i} g_{j}\right| \rho^{|i|+|j|}\right)_{(i, j) \in \mathbb{Z}^{2}}$ is a summable family. Using the triangular inequality and $\rho \geq 1$, we obtain that $\left|f_{i} g_{j}\right| \rho^{|i+j|}$ is a summable family, so the following computation is valid:

$$
\sum_{i \in \mathbb{Z}}\left|h_{i}\right| \rho^{|i|} \leq \sum_{(i, k) \in \mathbb{Z}^{2}}\left|f_{k} g_{i-k}\right| \rho^{|i|}=\sum_{(i, j) \in \mathbb{Z}^{2}}\left|f_{i} g_{j}\right| \rho^{|i+j|} \leq \sum_{i \in \mathbb{Z}}\left|f_{i}\right| \rho^{|i|} \sum_{j \in \mathbb{Z}}\left|g_{j}\right| \rho^{|j|} .
$$

Hence $h \in \mathcal{W}_{\rho}$ and $\|h\| \leq\|f\| \times\|g\|$.

Recall that if $L$ is a loop group, $L_{\rho} \subset L$ denotes the subgroup of loops whose entries are in the Banach algebra $\mathcal{W}_{\rho}$.

Proposition 8. Let $\Phi \in \Lambda S L(2, \mathbb{C})_{\rho}$ and $(F, B)$ be its Iwasawa decomposition. Then $F \in$ $\Lambda S U(2)_{\rho}$ and $B \in \Lambda_{+}^{\mathbb{R}} S L(2, \mathbb{C})_{\rho}$.

Proof: $\Phi$ extends holomorphically to the annulus $\frac{1}{\rho}<|\lambda|<\rho$ and $B$ extends holomorphically to the disk $|\lambda|<1$, so $F=\Phi B^{-1}$ extends holomorphically to the annulus $\frac{1}{\rho}<|\lambda|<1$. By an application of the Schwarz reflection principle, the fact that $F(\lambda) \in S U(2)$ on the unit circle implies that $F$ extends holomorphically to the annulus $\frac{1}{\rho}<|\lambda|<\rho$ and satisfies (see details in Appendix A of [11]):

$$
F_{11}\left(\frac{1}{\bar{\lambda}}\right)=\overline{F_{22}(\lambda)} \quad \text { and } \quad F_{12}\left(\frac{1}{\bar{\lambda}}\right)=-\overline{F_{21}(\lambda)} .
$$

We expand $F=\left(F_{k \ell}\right)_{1 \leq k, \ell \leq 2}$ in the annulus $\frac{1}{\rho}<|\lambda|<\rho$ as

$$
F(\lambda)=\sum_{i \in \mathbb{Z}} F_{i} \lambda^{i} \quad \text { with } \quad F_{i}=\left(F_{k \ell, i}\right)_{1 \leq k, \ell \leq 2} .
$$

Then for all $i \in \mathbb{Z}$

$$
F_{11,-i}=\overline{F_{22, i}} \quad \text { and } \quad F_{12,-i}=-\overline{F_{21, i}} .
$$

We expand $\Phi$ and $B^{-1}$ in the annulus $\frac{1}{\rho}<|\lambda|<1$ as

$$
\Phi(\lambda)=\sum_{i \in \mathbb{Z}} \Phi_{i} \lambda^{i}, \quad B^{-1}(\lambda)=\sum_{i \in \mathbb{Z}} C_{i} \lambda^{i}
$$

with $C_{i}=0$ for $i<0$. Then

$$
F_{i}=\sum_{j \in \mathbb{Z}} \Phi_{j} C_{i-j}
$$

Since $\Phi \in \Lambda S L(2, \mathbb{C})_{\rho},\left(\left\|\Phi_{i}\right\| \rho^{-i}\right)_{i \in \mathbb{Z}}$ is a summable family (here $\|A\|=\max \left|A_{k \ell}\right|$ for $A \in$ $\left.\mathcal{M}_{2}(\mathbb{C})\right)$. Since $B^{-1}$ is holomorphic in the unit disk, $\left(\left\|C_{i}\right\| \rho^{-i}\right)_{i \in \mathbb{Z}}$ is a summable family. Hence

$$
\sum_{i \in \mathbb{Z}}\left\|F_{i}\right\| \rho^{-i}<\infty
$$

By Equation (27), this implies

$$
\sum_{i \in \mathbb{Z}}\left\|F_{i}\right\| \rho^{i}<\infty
$$

Hence $F \in \mathcal{M}_{2}\left(\mathcal{W}_{\rho}\right)$. Since $\mathcal{W}_{\rho}$ is a Banach algebra, $B=F^{-1} \Phi \in \mathcal{M}_{2}\left(\mathcal{W}_{\rho}\right)$ as well.

Remark 4. If $\Phi \in \Lambda S L(2, \mathbb{C})$ is a Laurent polynomial of degree $d$, a similar argument proves that $F$ is a Laurent polynomial of degree at most $d$ and $B$ is a polynomial of degree at most $2 d$. 
The loop groups $\Lambda S L(2, \mathbb{C})_{\rho}, \Lambda S U(2)_{\rho}$ and $\Lambda_{+}^{\mathbb{R}} S L(2, \mathbb{C})_{\rho}$ are Banach manifolds. This can be proved using the submersion criterion (see [1], 5.9.1 for the definition of a submersion in the Banach case). The tangent space at $I_{2}$ of these Banach Lie groups are respectively the following Banach Lie algebras:

$$
\begin{aligned}
& \Lambda \mathfrak{s l}(2, \mathbb{C})_{\rho}=\left\{M \in \mathcal{M}_{2}\left(\mathcal{W}_{\rho}\right): M_{11}+M_{22}=0\right\} \\
& \Lambda \mathfrak{s u}(2)_{\rho}=\left\{M \in \mathcal{M}_{2}\left(\mathcal{W}_{\rho}\right): M_{11}+M_{22}=0, M_{11}+M_{11}^{*}=0, M_{12}+M_{21}^{*}=0\right\} \\
& \Lambda_{+}^{\mathbb{R}} \mathfrak{s l}(2, \mathbb{C})_{\rho}=\left\{M \in \mathcal{M}_{2}\left(\mathcal{W}_{\rho}^{\geq 0}\right): M_{11}+M_{22}=0, M_{11}(0) \in \mathbb{R}, M_{21}(0)=0\right\} .
\end{aligned}
$$

\section{Proposition 9.}

$$
\Lambda \mathfrak{s l}(2, \mathbb{C})_{\rho}=\Lambda \mathfrak{s u}(2)_{\rho} \oplus \Lambda_{+}^{\mathbb{R}} \mathfrak{s l}(2, \mathbb{C})_{\rho}
$$

Proof:

- Let $M \in \Lambda \mathfrak{s u}(2)_{\rho} \cap \Lambda_{+}^{\mathbb{R}} \mathfrak{s l}(2, \mathbb{C})_{\rho}$. Then all entries of $M$ are in $\mathcal{W}_{\rho}^{\geq 0} \cap \mathcal{W}_{\rho}^{\leq 0}=\mathcal{W}^{0}$ so $M$ is constant. It is then straightforward that $M=0$.

- Let $M \in \Lambda \mathfrak{s l}(2, \mathbb{C})_{\rho}$ and write

$$
M(\lambda)=\sum_{n \in \mathbb{Z}} M_{n} \lambda^{n} \quad \text { with } \quad M_{0}=\left(\begin{array}{cc}
a_{0} & b_{0} \\
c_{0} & -a_{0}
\end{array}\right) .
$$

Define

$$
\begin{gathered}
\operatorname{uni}(M)(\lambda)=\left(\begin{array}{cc}
\mathrm{i} \operatorname{Im}\left(a_{0}\right) & -\overline{c_{0}} \\
c_{0} & -\mathrm{i} \operatorname{Im}\left(a_{0}\right)
\end{array}\right)+\sum_{n<0}\left(M_{n} \lambda^{n}-{ }^{t} \bar{M}_{n} \lambda^{-n}\right) \in \Lambda \mathfrak{s u}(2)_{\rho} \\
\operatorname{pos}(M)(\lambda)=\left(\begin{array}{cc}
\operatorname{Re}\left(a_{0}\right) & b_{0}+\overline{c_{0}} \\
0 & -\operatorname{Re}\left(a_{0}\right)
\end{array}\right)+\sum_{n>0} M_{n} \lambda^{n}+\sum_{n<0}{ }^{t} \bar{M}_{n} \lambda^{-n} \in \Lambda_{+}^{\mathbb{R}} \mathfrak{s l}(2, \mathbb{C})_{\rho} .
\end{gathered}
$$

Then $M=\operatorname{uni}(M)+\operatorname{pos}(M)$.

Theorem 5. Iwasawa decomposition is a smooth diffeomorphism (in the sense of Banach manifolds) from $\Lambda S L(2, \mathbb{C})_{\rho}$ to $\Lambda S U(2)_{\rho} \times \Lambda_{+}^{\mathbb{R}} S L(2, \mathbb{C})_{\rho}$. Moreover, its differential at $I_{2}$ is given by $d \mathrm{Uni}\left(I_{2}\right)=$ uni and $d \operatorname{Pos}\left(I_{2}\right)=$ pos.

Proof: consider the following smooth map:

$$
p: \Lambda S U(2)_{\rho} \times \Lambda_{+}^{\mathbb{R}} S L(2, \mathbb{C})_{\rho} \rightarrow \Lambda S L(2, \mathbb{C})_{\rho}, \quad p(F, B)=F B .
$$

Then

$$
d p\left(I_{2}, I_{2}\right): \Lambda \mathfrak{s u}(2)_{\rho} \times \Lambda_{+}^{\mathbb{R}} \mathfrak{s l}(2, \mathbb{C})_{\rho} \rightarrow \Lambda \mathfrak{s l}(2, \mathbb{C})_{\rho}, \quad d p\left(I_{2}, I_{2}\right)(F, B)=F+B .
$$

By Proposition 9, $d p\left(I_{2}, I_{2}\right)$ is an isomorphism. By the Inverse Mapping Theorem, $p$ is a local diffeomorphism in a neighborhood of $\left(I_{2}, I_{2}\right)$. Let $\left(F_{0}, B_{0}\right) \in \Lambda S U(2)_{\rho} \times \Lambda_{+}^{\mathbb{R}} S L(2, \mathbb{C})_{\rho}$. Consider the maps

$$
\begin{gathered}
f_{1}: \Lambda S U(2)_{\rho} \times \Lambda_{+}^{\mathbb{R}} S L(2, \mathbb{C})_{\rho} \rightarrow \Lambda S U(2)_{\rho} \times \Lambda_{+}^{\mathbb{R}} S L(2, \mathbb{C})_{\rho}, \quad f_{1}(F, B)=\left(F_{0} F, B B_{0}\right) \\
f_{2}: \Lambda S L(2, \mathbb{C})_{\rho} \rightarrow \Lambda S L(2, \mathbb{C})_{\rho}, \quad f_{2}(\Phi)=F_{0} \Phi B_{0} .
\end{gathered}
$$

Then $f_{1}$ is a diffeomorphism with inverse $(F, B) \mapsto\left(F_{0}^{-1} F, B B_{0}^{-1}\right)$ and $f_{2}$ is a diffeomorphism with inverse $\Phi \mapsto F_{0}^{-1} \Phi B_{0}^{-1}$. We have

$$
f_{2} \circ p \circ f_{1}^{-1}=p
$$


so $p$ is a local diffeomorphism in a neighborhood of any $\left(F_{0}, B_{0}\right)$. Since $p$ is injective (by the standard Iwasawa decomposition theorem) and onto (by Proposition 8), $p$ is a smooth diffeomorphism. Iwasawa decomposition is of course the inverse of $p$.

As an application of Theorem 5, let us prove Equation (8) by differentiation of the SymBobenko formula (6). In the following computation, we omit the $\lambda$ variable. Fix $z_{0} \in \Sigma$ and define $\widetilde{\Phi}(z)=F\left(z_{0}\right)^{-1} \Phi(z) B\left(z_{0}\right)^{-1}$. Then

$$
d \widetilde{\Phi}\left(z_{0}\right)=F\left(z_{0}\right)^{-1} \Phi\left(z_{0}\right) \xi\left(z_{0}\right) B\left(z_{0}\right)^{-1}=B\left(z_{0}\right) \xi\left(z_{0}\right) B\left(z_{0}\right)^{-1} .
$$

Writing $B\left(z_{0}\right)^{0}=\left(\begin{array}{cc}\rho & \mu \\ 0 & \rho^{-1}\end{array}\right)$ and $\xi_{12}\left(z_{0}\right)=\lambda^{-1} \beta$, we have

$$
d \widetilde{\Phi}\left(z_{0}\right)^{-}=\left(\begin{array}{cc}
\rho & \mu \\
0 & \rho^{-1}
\end{array}\right)\left(\begin{array}{cc}
0 & \lambda^{-1} \beta^{0} \\
0 & 0
\end{array}\right)\left(\begin{array}{cc}
\rho^{-1} & -\mu \\
0 & \rho
\end{array}\right)=\left(\begin{array}{cc}
0 & \rho^{2} \lambda^{-1} \beta^{0} \\
0 & 0
\end{array}\right) .
$$

Since $\widetilde{\Phi}\left(z_{0}\right)=I_{2}$, Theorem 5 yields

$$
\begin{gathered}
F\left(z_{0}\right)^{-1} d F\left(z_{0}\right)=d \widetilde{F}\left(z_{0}\right)=\operatorname{uni}\left(d \widetilde{\Phi}\left(z_{0}\right)\right)=\left(\begin{array}{cc}
0 & \rho^{2} \lambda^{-1} \beta^{0} \\
-\rho^{2} \lambda \overline{\beta^{0}} & 0
\end{array}\right)+\text { constant } \\
\left.\frac{\partial}{\partial \lambda}\left(F\left(z_{0}\right)^{-1} d F\left(z_{0}\right)\right)\right|_{\lambda=1}=\left(\begin{array}{cc}
0 & -\rho^{2} \beta^{0} \\
-\rho^{2} \overline{\beta^{0}} & 0
\end{array}\right) .
\end{gathered}
$$

By differentiation of the Sym-Bobenko formula, we obtain (omitting $z_{0}$ )

$$
\begin{aligned}
d f & =-\left.2 \mathrm{i}\left(\frac{\partial d F}{\partial \lambda} F^{-1}-\frac{\partial F}{\partial \lambda} F^{-1} d F F^{-1}\right)\right|_{\lambda=1} \\
& =-\left.2 \mathrm{i} F \frac{\partial}{\partial \lambda}\left(F^{-1} d F\right) F^{-1}\right|_{\lambda=1} \\
& =\left.2 \mathrm{i} \rho^{2} F\left(\begin{array}{cc}
0 & \beta^{0} \\
\beta^{0} & 0
\end{array}\right) F^{-1}\right|_{\lambda=1} .
\end{aligned}
$$

Appendix B. Appendix: On Delaunay ends in the DPW method

We consider the standard Delaunay residue for $t \leq \frac{1}{16}$ :

$$
A_{t}(\lambda)=\left(\begin{array}{cc}
0 & \lambda^{-1} r+s \\
\lambda r+s & 0
\end{array}\right) \quad \text { where }\left\{\begin{array}{l}
r+s=\frac{1}{2} \\
r s=t \\
r<s
\end{array}\right.
$$

In particular, in the limit case $t=0$, we have

$$
A_{0}=\left(\begin{array}{cc}
0 & \frac{1}{2} \\
\frac{1}{2} & 0
\end{array}\right) .
$$

Let $\mathbb{A}_{\rho}$ be the annulus $\frac{1}{\rho}<|\lambda|<\rho$, where $\rho>1$.

Definition $6([1])$. A perturbed Delaunay potential is a family of DPW potentials $\xi_{t}$ of the form

$$
\xi_{t}(z, \lambda)=A_{t}(\lambda) \frac{d z}{z}+R_{t}(z, \lambda) d z
$$

where $R_{t}$ is of class $C^{2}$ with respect to $(t, z, \lambda) \in(-T, T) \times D(0, \varepsilon) \times \mathbb{A}_{\rho}$ for some positive $\varepsilon$ and $T$, and satisfies $R_{0}=0$. In particular, $\xi_{0}=A_{0} \frac{d z}{z}$. 
Let $\left(e_{1}, e_{2}, e_{3}\right)$ represent the canonical basis of $\mathbb{R}^{3}$ in the $\mathfrak{s u}(2)$ model.

Theorem 6. Let $\xi_{t}$ be a perturbed Delaunay potential. Let $\Phi_{t}(z, \lambda)$ be a family of solutions of $d \Phi_{t}=\Phi_{t} \xi_{t}$ in the universal cover of the punctured disk $D^{*}(0, \varepsilon)$. Assume that $\Phi_{t}(z, \lambda)$ depends continuously on $(t, z, \lambda)$ and that the Monodromy Problem for $\Phi_{t}$ is solved. Let $f_{t}=$ $\operatorname{Sym}\left(\operatorname{Uni}\left(\Phi_{t}\right)\right)$ be the immersion given by the DPW method. Finally, assume that $\Phi_{0}(1, \cdot)$ is constant (i.e. independent of $\lambda$ ).

Given $0<\alpha<1$, there exists uniform positive numbers $\varepsilon^{\prime} \leq \varepsilon, T^{\prime} \leq T$, $c$ and a family of Delaunay immersions $f_{t}^{\mathcal{D}}: \mathbb{C}^{*} \rightarrow \mathbb{R}^{3}$ such that:

(1) For $0<|t|<T^{\prime}$ and $0<|z|<\varepsilon^{\prime}$ :

$$
\left\|f_{t}(z)-f_{t}^{\mathcal{D}}(z)\right\| \leq c|t||z|^{\alpha} .
$$

(2) For $0<t<T^{\prime}, f_{t}: D^{*}\left(0, \varepsilon^{\prime}\right) \rightarrow \mathbb{R}^{3}$ is an embedding.

(3) The end of $f_{t}^{\mathcal{D}}$ at $z=0$ has weight $8 \pi t$ and its axis converges when $t \rightarrow 0$ to the half-line spanned by the vector $Q e_{3} Q^{-1}$ where

$$
Q=\operatorname{Uni}\left(\Phi_{0}(1) H\right) \quad \text { and } \quad H=\frac{1}{\sqrt{2}}\left(\begin{array}{cc}
1 & 1 \\
-1 & 1
\end{array}\right)
$$

Thomas Raujouan has proved this result in [11, Theorem 3, in the case $\Phi_{0}(1, \lambda)=I_{2}$. He proves that the limit axis is spanned by $e_{1}$. (In fact, he finds that the limit axis is $-e_{1}$, but this is because he has the opposite sign in the Sym-Bobenko formula. See Remark 2, ) Then in Section 2 of [11, he explains, in the case $r>s$, how to extend his result to the case where $\Phi_{0}(1, \lambda)$ is constant. We adapt his method to the case $r<s$.

Lemma 2. There exists a gauge $G(z)$ and a change of variable $h(z)$ with $h(0)=0$ such that $\widetilde{\xi}_{t}=\left(h^{*} \xi_{t}\right) \cdot G$ is a perturbed Delaunay potential (with residue $A_{t}$ ) and $\widetilde{\Phi}_{t}=\left(h^{*} \Phi_{t}\right) \times G$ satisfies at $t=0$

$$
\widetilde{\Phi}_{0}(1, \lambda)=Q H^{-1} \in \Lambda S U(2) .
$$

Proof: we follow the method explained in Section 2 of [11]. We take the change of variable in the form

$$
h(z)=\frac{z}{p z+q}
$$

where $p, q$ are complex numbers (independent of $t$ ) to be determined, with $q \neq 0$. We consider the following gauge:

$$
G(z)=\frac{1}{\sqrt{q(p z+q)}}\left(\begin{array}{cc}
p z+q & p z \\
0 & q
\end{array}\right) .
$$

It is chosen so that

$$
G(0)=I_{2} \quad \text { and } \quad d G=G A_{0} \frac{d z}{z}-A_{0} G \frac{d h}{h} .
$$

(In fact, the gauge $G$ is found as the only solution of Problem 29 which is upper triangular.) We have

$$
\widetilde{\xi}_{t}=\left(h^{*} \xi_{t}\right) \cdot G=G^{-1}\left(A_{t}(\lambda) \frac{d h}{h}+R_{t}(h, \lambda) d h\right) G+G^{-1} d G .
$$


Since $G(0)=I_{2}, \widetilde{\xi}_{t}$ has a simple pole at $z=0$ with residue $A_{t}$. Using Equation (29), we obtain at $t=0$ :

$$
\widetilde{\xi}_{0}=G^{-1} A_{0} \frac{d h}{h} G+G^{-1} d G=A_{0} \frac{d z}{z} .
$$

Hence $\widetilde{\xi}_{t}$ is a perturbed Delaunay potential. It remains to compute $\widetilde{\Phi}_{0}(1)$. The matrix $H$ diagonalises $A_{0}$ :

$$
A_{0}=H\left(\begin{array}{cc}
\frac{-1}{2} & 0 \\
0 & \frac{1}{2}
\end{array}\right) H^{-1}
$$

Hence

$$
\begin{aligned}
& \Phi_{0}(z)=\Phi_{0}(1) z^{A_{0}}=\Phi_{0}(1) H\left(\begin{array}{cc}
\frac{1}{\sqrt{z}} & 0 \\
0 & \sqrt{z}
\end{array}\right) H^{-1} \\
\widetilde{\Phi}_{0}(1)= & \Phi_{0}(h(1)) G(1) \\
= & \Phi_{0}(1) H\left(\begin{array}{cc}
\sqrt{p+q} & 0 \\
0 & \frac{1}{\sqrt{p+q}}
\end{array}\right) H^{-1} \frac{1}{\sqrt{q(p+q)}}\left(\begin{array}{cc}
p+q & p \\
0 & q
\end{array}\right) \\
= & \Phi_{0}(1) H\left(\begin{array}{cc}
\sqrt{q} & \frac{p}{\sqrt{q}} \\
0 & \frac{1}{\sqrt{q}}
\end{array}\right) H^{-1}
\end{aligned}
$$

We decompose $\Phi_{0}(1) H=Q R$ with $Q \in S U(2)$ and $R=\left(\begin{array}{cc}\rho & \mu \\ 0 & \frac{1}{\rho}\end{array}\right)$. Then

$$
\widetilde{\Phi}_{0}(1)=Q\left(\begin{array}{cc}
\rho & \mu \\
0 & \frac{1}{\rho}
\end{array}\right)\left(\begin{array}{cc}
\sqrt{q} & \frac{p}{\sqrt{q}} \\
0 & \frac{1}{\sqrt{q}}
\end{array}\right) H^{-1}
$$

We take $q=\frac{1}{\rho^{2}}$ and $p=-\frac{\mu}{\rho}$ to cancel the two matrices in the middle and obtain Equation 28,

We can now prove Theorem 6. Let

$$
\widehat{\Phi}_{t}(z, \lambda)=H Q^{-1} \widetilde{\Phi}_{t}(z, \lambda)=H Q^{-1} \Phi_{t}(h(z), \lambda) G(z, \lambda)
$$

Since $\widehat{\Phi}_{0}(1, \lambda)=I_{2}$, we can apply Theorem 3 in [1] which says that the resulting immersion $\widehat{f}_{t}$ satisfies Points 1 and 2 of Theorem 6 and its limit axis is spanned by $e_{1}$. We have

$$
f_{t} \circ h=Q H^{-1} \widehat{f}_{t} H Q^{-1}
$$

so $f_{t} \circ h$ and $\widehat{f_{t}}$ differ by a rotation and the limit axis of $f_{t}$ is spanned by the vector $Q H^{-1} e_{1} H Q^{-1}$. Now

$$
H^{-1} e_{1} H=-\frac{\mathrm{i}}{2}\left(\begin{array}{cc}
1 & -1 \\
1 & 1
\end{array}\right)\left(\begin{array}{ll}
0 & 1 \\
1 & 0
\end{array}\right)\left(\begin{array}{cc}
1 & 1 \\
-1 & 1
\end{array}\right)=-\mathrm{i}\left(\begin{array}{cc}
-1 & 0 \\
0 & 1
\end{array}\right)=e_{3}
$$




\section{REFERENCES}

[1] N. Bourbaki: Variétés différentielles et analytiques. Eléments de mathématiques. Hermann, Paris 1971.

[2] C. Cosín, A. Ros: A Plateau problem at infinity for properly immersed minimal surfaces with finite total curvature. Indiana Univ. Math. J. 50, no. 2 (2001), 847-879.

[3] J. Dorfmeister, F. Pedit, H. Wu: Weierstrass type representation of harmonic maps into symmetric spaces. Communications in Analysis and Geometry 6 (1998), 633-668.

[4] O. Forster: Lectures on Riemann surfaces. Graduate texts in Mathematics, Springer Verlag (1981).

[5] S. Fujimori, S. Kobayashi, W. Rossman: Loop group methods for constant mean curvature surfaces. arXiv:math/0602570

[6] K. Große-Brauckmann, R. Kusner, J. Sullivan: Triunduloids: embedded constant mean curvature surfaces with three ends and genus zero. J. Reine Angew. Math. 564 (2003), 35-61.

[7] K. Große-Brauckmann, R. Kusner, J. Sullivan: Coplanar constant mean curvature surfaces. Comm. Anal. Geom. 15 (2007), no. 5, 985-1023.

[8] N. Kapouleas: Complete constant mean curvature surfaces in euclidean three-space. Annals of Mathematics 131 (1990), 239-330.

[9] M. Kilian, W. Rossman, N. Schmitt: Delaunay ends of constant mean curvature surfaces. Compositio Mathematica 144 (2008), 186-220.

[10] R. Mazzeo, F. Pacard: Constant mean curvature surfaces with Delaunay ends. Comm. Anal. and Geom. 9, no. 1 (2001), 169-237.

[11] T. Raujouan: On Delaunay ends in the DPW method. To appear in Indiana Univ. Math. J. arXiv:1710.00768v2 (2017).

[12] N. Schmitt: Constant mean curvature trinoids. arXiv:math/0403036 (2004).

[13] N. Schmitt, M. Kilian, S. Kobayashi, W. Rossman: Unitarization of monodromy representations and constant mean curvature trinoids in 3-dimensional space forms. Journal of the London Mathematical Society 75 (2007), 563-581.

[14] M. Traizet: Construction of constant mean curvature n-noids using the DPW method. To appear in Journal für die reine und angewandte Mathematik. arXiv 1709.00924 (2017).

[15] M. Traizet: Opening nodes in the DPW method. arXiv 1808.01366 (2018).

Martin Traizet

Institut Denis Poisson

Université de Tours, 37200 Tours, France

martin.traizet@univ-tours.fr 